\title{
Axon-secreted chemokine-like Orion is a signal for astrocyte infiltration during neuronal remodeling
}

Ana Boulanger ${ }^{1,}$, , Camille Thinat ${ }^{1}$, Stephan Züchner ${ }^{2}$, Lee G. Fradkin ${ }^{3}$, Hugues Lortat-Jacob ${ }^{4}$ and Jean-Maurice Dura ${ }^{1,5, *}$

${ }^{1}$ IGH, Centre National de la Recherche Scientifique, Univ Montpellier, Montpellier, France

${ }^{2}$ Dr. John T. Macdonald Foundation Department of Human Genetics and John P. Hussman Institute for Human Genomics, University of Miami, Miami, FL, USA.

${ }^{3}$ Department of Neurobiology, University of Massachusetts Medical School, 364 Plantation Ave. Worcester, MA 01655, USA. Present contact: leeffradkin@gmail.com (1)

${ }^{4}$ Université Grenoble Alpes, Centre National de la Recherche Scientifique, and Commissariat à l'Énergie Atomique et aux Énergies Alternatives, Institut de Biologie Structurale, UMR 5075, Grenoble, France

\footnotetext{
${ }^{5}$ Lead Contact
}

*Correspondence: ana.boulanger@igh.cnrs.fr; jean-maurice.dura@igh.cnrs.fr

Short title: Neuron-glial crosstalk for axon removal 


\section{Summary}

The remodeling of neurons is a conserved fundamental mechanism underlying nervous system maturation and function. Glial cells are known to clear neuronal debris but also to have an active role in the remodeling process. Developmental axon pruning of Drosophila memory center neurons occurs by a degenerative process mediated by infiltrating astrocytes. However, how these glial processes are recruited by the axons is unknown. In an unbiased screen, we identified a new gene (orion) which is necessary for both the pruning of some axons and removal of the resulting debris. Orion is secreted from the neurons and bears some features common to the chemokines, a family of chemoattractant cytokines. Thus, chemokine involvement in neuron/glial cell interaction is an evolutionarily ancient mechanism. We propose that Orion is the neuronal signal that elicits astrocyte infiltration required for developmental neuronal remodeling.

\section{Introduction}

Neuronal remodeling is a widely used developmental mechanism, across the animal kingdom, to refine dendrite and axon targeting necessary for the maturation of neural circuits. Importantly, similar molecular and cellular events can occur during neurodevelopmental disorders or after nervous system injury ${ }^{1-4}$. A key role for glial cells in synaptic pruning and critical signaling pathways between glia and neurons have been identified ${ }^{4}$. In Drosophila, the mushroom body (MB), a brain memory center, is remodeled at metamorphosis and $\mathrm{MB} \gamma$ neuron pruning occurs by a degenerative mechanism ${ }^{5-8}$. Astrocytes surrounding the MB have an active role in the process; blocking their infiltration into the MBs prevents remodeling ${ }^{9-12}$. MB $\gamma$ neuron remodeling relies on two processes: axon fragmentation and the subsequent clearance of axonal debris. Importantly, it has been shown that astrocytes are involved in these two processes and that these two processes can be decoupled ${ }^{12}$. Altering the ecdysone signaling in astrocytes, during metamorphosis, results both in a partial axon pruning defect, visualized as either some individual larval axons or as thin bundles of intact larval axons remaining in the adults, and also in a strong defect in clearance of debris, visualized by the presence of clusters of axonal debris. Astrocytes have only a minor role in axon severing as evidenced by the observation that most of the MB $\gamma$ axons are correctly pruned when ecdysone signaling is altered in these cells. When astrocyte function is blocked, the $\gamma$ axon-intrinsic fragmentation process remains functional and the majority of axons degenerate. 
It has been widely proposed that a "find-me/eat-me" signal emanating from the degenerating $\gamma$ neurons is necessary for astrocyte infiltration ${ }^{7,9,13}$. However, the nature of this glial recruitment signal is unknown.

Here we have identified a new gene (orion) by screening for viable ethyl methanesulfonate (EMS)-induced mutations and not for lethal mutations in MB clones as was done previously ${ }^{14,15}$. This allowed the identification of genes involved in glia cell function by directly screening for defects in MB axon pruning. We found that orion $^{l}$, a viable $\mathrm{X}$ chromosome mutation, is necessary for both the pruning of some $\gamma$ axons and removal of the resulting debris. We show that Orion is secreted from the neurons, remains near the axon membranes where it associates with infiltrating astrocytes, and is necessary for astrocyte infiltration into the $\gamma$ bundle. This implies a role for an as-yet-undefined Orion receptor on the surface of the astrocytes. Orion bears some chemokine features, e.g, a $\mathrm{CX}_{3} \mathrm{C}$ motif, 3 glycosaminoglycan binding consensus sequences that are required for its function. Altogether, our results identify a neuron-secreted extracellular messenger, which is likely to be the longsearched-for signal responsible for astrocyte infiltration and demonstrate its involvement for neuronal remodeling.

\section{Results and Discussion}

\section{The orion gene is necessary for MB remodeling}

Adult orion $^{l}$ individuals showed a clear and highly penetrant MB axon pruning phenotype as revealed by the presence of some adult unpruned vertical $\gamma$ axons as well as the strong presence of debris $(100 \%$ of mutant MBs; $n=100)$ (Fig. 1a, b, Table I and Supplementary Fig.1 and 2). Astrocytes, visualized with alrm-GAL4, are the major glial subtype responsible for the clearance of the MB axon debris ${ }^{12}$. The presence of $\gamma$ axon debris is a landmark of defective astrocyte function, as was previously described ${ }^{11,12}$, and is also further shown in this study (Supplementary Fig. 1a-d). The unpruned axon phenotype was particularly apparent during metamorphosis (Fig. 1c-h). At $24 \mathrm{~h}$ after puparium formation (APF), although $\gamma$ axon branches were nearly completely absent in the wild-type control they persisted in the orion ${ }^{l}$ mutant brains, where we also observed a significant accumulation of debris (Fig. 1e, h). The number of unpruned axons at this stage is lower in orion $^{1}$ than in $\mathrm{Hr} 39^{C 13}$ where the $\gamma$ axon-intrinsic process of pruning is blocked (Supplementary Fig. 1 e-g). In addition, the MB dendrite pruning was clearly affected in orion $^{l}$ individuals (Supplementary Fig. 1h-p).

\section{The orion gene encodes for a $\mathrm{CX}_{3} \mathrm{C}$ motif-containing secreted proteins}




\section{1}

The orion $^{l}$ EMS mutation was localized by standard duplication and deficiency mapping as well as by whole genome sequencing (Fig. 2a). The orion gene (CG2206) encodes two putatively secreted proteins: Orion-A (664 aa) and Orion-B (646 aa), whose mRNAs arise from two different promoters (Fig. 2b-d). We have confirmed that both mRNAs are present in early pupal brains by RT-PCR (data not shown). These two proteins differ in their N-terminal domains and are identical in the remainder of their sequences. The EMS mutation is a $\mathrm{G}$ to $\mathrm{C}$ nucleotide change inducing the substitution of the glycine (at position 629 for Orion-A and 611 for Orion-B) into an aspartic acid. The mutation lies in the common shared part and therefore affects both Orion-A and -B functions. Both isoforms display a signal peptide at their N-termini suggesting that they are secreted. Interestingly, a $\mathrm{CX}_{3} \mathrm{C}$ chemokine signature is present in the Orion common region (Fig. 2b, c). Chemokines are a family of chemoattractant cytokines, characterized by a $\mathrm{CC}, \mathrm{CXC}$ or $\mathrm{CX}_{3} \mathrm{C}$ motif, promoting the directional migration of cells within different tissues. Mammalian $\mathrm{CX}_{3} \mathrm{CL} 1$ (also known as fractalkine) is involved in, among other contexts, neuron-glia communication ${ }^{16-20}$. Mammalian Fractalkines display conserved intramolecular disulfide bonds that appear be conserved with respect to their distance from the $\mathrm{CX}_{3} \mathrm{C}$ motif present in both Orion isoforms (Fig. 2c). Fractalkine and its receptor, $\mathrm{CX}_{3} \mathrm{CR} 1$, have been recently shown to be required for post-trauma cortical brain neuron microgliamediated remodeling in a mouse whisker lesioning paradigm ${ }^{21}$. We observed that the change of the $\mathrm{CX}_{3} \mathrm{C}$ motif into $\mathrm{CX}_{4} \mathrm{C}$ or $\mathrm{AX}_{3} \mathrm{C}$ blocked the Orion function necessary for the $\mathrm{MB}$ pruning (Supplementary Fig. 3a-c, h-j). Similarly, the removal of the signal peptide also prevented pruning (Supplementary Fig. 3d, h-j). These two results indicate that the Orion isoforms likely act as secreted chemokine-like molecules. We also produced three CRISPR/Cas9-mediated mutations in the orion gene, which either delete the common part $\left(\right.$ orion $\left.^{\Delta C}\right)$, the A-specific part $\left(\right.$ orion $\left.^{\Delta A}\right)$ or the B-specific part $\left(\right.$ orion $\left.^{\Delta B}\right)$. Noticeably, orion ${ }^{\Delta C}$ displayed the same MB pruning phenotype as orion $^{l}$ which is also the same in orion ${ }^{l} /$ Deficiency females indicating that orion $^{l}$ and orion $^{\Delta C}$ are likely null alleles for this phenotype. In contrast, orion $^{\triangle A}$ and orion $^{\Delta B}$ have no MB phenotype by themselves indicating the likelihood of functional redundancy between the two proteins in the pruning process (Supplementary Fig. 4).

\section{Orion is required and expressed by MB $\gamma$ axons}

Using the GAL4/UAS system ${ }^{22}$, we found that expression of wild-type orion in the orion $^{1} \mathrm{MB}$ $\gamma$ neurons (201Y-GAL4) fully rescued the MB mutant phenotype $(100 \%$ of wild-type MBs $\mathrm{n}=$ 387; see quantitation in Supplementary Fig. 3h) although wild-type orion expression in the astrocytes (alrm-GAL4) did not rescue (Fig. 1i-k and Supplementary Fig. 5a-c). repo-GAL4 
could not be used because of lethality when combined with UAS-orion. This supports the hypothesis that Orion is produced by axons and, although necessary for astrocyte infiltration, not by astrocytes. Both $U A S$-orion- $A$ and $U A S$-orion- $B$ rescued the orion ${ }^{l}$ pruning phenotype indicating again a likely functional redundancy between the two proteins at least in the pruning process. Complementary to the rescue results, we found that the expression of an oriontargeting RNAi in the MBs produced unpruned axons similar to that in orion ${ }^{1}$ although debris are not apparent likely due to an incomplete inactivation of the gene expression by the RNAi (Fig. 11 and Supplementary Fig. 5d). The expression of the same RNAi in the glia had no effect (Supplementary Fig. 5e). Using the mosaic analysis with a repressible cell marker (MARCM ${ }^{23}$ ), we found that orion ${ }^{1}$ homozygous mutant neuroblast clones of $\gamma$ neurons, in orion $^{1 /+}$ phenotypically wild-type individuals, were normally pruned (Supplementary Fig. 6a, b). Therefore, orion $^{l}$ is a non-cell-autonomous mutation which is expected since the Orion proteins are secreted (see below). Orion proteins secreted by the surrounding wild-type axons rescue the pruning defects in the orion mutant clones.

From our genetic data, orion expression is expected in the $\gamma$ neurons. The fine temporal transcriptional landscape of MB $\gamma$ neurons was recently described and a corresponding resource is freely accessible ${ }^{24}$. Noteworthy, orion is transcribed at $0 \mathrm{~h}$ APF and dramatically decreases at 9h APF with a peak at $3 \mathrm{~h}$ APF (Supplementary Fig. 7). The nuclear receptors EcR-B1 and its target Sox 14 are key transcriptional factors required for MB neuronal remodeling ${ }^{6,7}$. orion was found to be a likely transcriptional target of EcR-B1 and Sox $14{ }^{24}$ and this is also consistent with earlier microarray analysis observations ${ }^{25}$. Noticeably, forced expression of $U A S-E c R-B 1$ in the MBs did not rescue the orion mutant phenotype and EcR-B1 expression, in the MB nuclei, is not altered in orion ${ }^{l}$ individuals (Supplementary Fig. 6c, f). Furthermore, the unpruned axon phenotype produced by orion RNAi is rescued by forced expression of $E c R-B 1$ in the MBs (Supplementary Fig. 3h). Therefore, our genetic interaction analyses support orion being downstream of $E c R-B 1$.

\section{Orion is secreted by MB $\gamma$ axons}

We focused our further molecular and cellular work on Orion-B alone since a functional redundancy between the two isoforms was apparent. We expressed the Orion-B protein in the $\gamma$ neurons using an UAS-orion-B-Myc insert and the $201 Y$-GAL4 driver. Orion-B was seen along the MB lobes and at short distances away from the axons as visualized by anti-Myc staining (Fig. 3). In addition, anti-Myc staining was particularly clear at the tip of the lobes indicating the secretion of Orion-B (Fig. 3a, d, g, j, k). Synaptic terminals are condensed in the $\gamma$ axon 
160 varicosities that disappear progressively during remodeling and hole-like structures

161 corresponding to the vestiges of disappeared varicosities can be observed at $6 \mathrm{~h} \mathrm{APF}^{9}$. We noted

162 the presence of secreted Myc-labelled Orion-B inside these hole-like structures (Fig. 3b, e, h).

163 The secretion of the Orion proteins should be under the control of their signal peptide and

164 therefore, Orion proteins lacking their signal peptide ( $\triangle \mathrm{SP})$ should not show this "secretion"

165 phenotype. When $U A S$-orion-B-Myc- $\triangle S P$ was expressed, Orion-B was not observed outside of

166 the axons or in the hole-like structures (Fig. 3c, f, i). We also excluded the possibility that this

167 "secretion" phenotype was due to some peculiarities of the Myc labelling by using a UAS-drl-

$168 M y c$ construct $^{26}$. Drl is a membrane-bound receptor tyrosine kinase and Drl-Myc staining,

169 unlike Orion-B, was not observed outside of the axons or in the hole-like structures

170 (Supplementary Fig. 6g-1). Finally, the presence of Myc-labelled Orion-B secreted protein not

171 associated with GFP-labelled axon membranes can be observed outside of the $\gamma$ axon bundle in

172 3D reconstructing images (Fig. 3j, k).

\section{Orion is required for the infiltration of astrocytes into the MB $\gamma$ bundle}

175 Since glial cells are likely directly involved in the orion $^{l}$ pruning phenotype, we examined their behavior early during the pruning process. At $6 \mathrm{~h}$ APF the axon pruning process starts and is complete by $24 \mathrm{~h}$ APF but the presence of glial cells in the vicinity of the wild-type $\gamma$ lobes is already clearly apparent at $6 \mathrm{~h} \mathrm{APF}^{9}$. We examined glial cells visualized by a membranetargeted GFP (UAS-mGFP) under the control of repo-GAL4 and co-stained the $\gamma$ axons with anti-Fas2. At $6 \mathrm{~h}$ APF a striking difference was noted between wild-type and orion $^{1}$ brains.

181 Unlike in the wild-type control, there is essentially no glial cell invasion of the $\gamma$ bundle in the mutant (Fig. 4a-c). Interestingly, glial infiltration was not observed in orion $^{1}$ neither at $12 \mathrm{~h}$ APF nor at 24 h APF (Supplementary Fig. 8 a-h) suggesting that glial cells never infiltrate MBs in mutant individuals. We also ruled out the possibility that this lack of glial cell infiltration was due to a lower number of astrocytes in mutant versus wild-type brains (Supplementary Fig. $8 \mathrm{i}, \mathrm{j})$.

We also examined the proximity between MB-secreted Orion-Myc and astrocytes, as inferred from the shape of the glial cells, labelled with the anti-Drpr antibody at $6 \mathrm{~h}$ APF (Fig. 4d-f). We looked at the distribution along the vertical $\gamma$ lobes ( $60 \mu \mathrm{m}$ of distance, see Methods) of Orion-B-Myc (secreted) and of Orion-B- $\Delta$ SP-Myc (not secreted), in an otherwise wild-type background. We quantified only from images where an astrocyte sat on the top of the vertical lobe. A peak of Orion-Myc localization was always found $(n=10)$ in the region close to the astrocyte (less than $7 \mu \mathrm{m}$ ) when secreted Orion-B-Myc was quantified (Fig. 4g, i). However, 
194 this was not the case $(n=9)$ when Orion-B- $\Delta$ SP-Myc was quantified (Fig. $4 \mathrm{~h}, \mathrm{j})$. This strongly suggests that astrocytic processes may be "attracted" by secreted Orion.

Moreover, we observed that secreted Orion stays close to axon membranes (Supplementary Fig. 9a-f). Protein, in particular chemokine, localization to membranes is often mediated by glycosaminoglycans (GAGs), a family of highly anionic polysaccharides that occur both at the cell surface and within the extracellular matrix. GAGs, to which all chemokines bind, ensure that these signaling proteins are presented at the correct site and time in order to mediate their functions ${ }^{27}$. We identified three consensus sequences for GAG linkage in the common part of Orion (Fig. 2d). We mutated these sequences individually and assayed the mutant proteins for their ability to rescue the orion ${ }^{l}$ pruning deficit in vivo. The three GAG sites are required for full Orion function, although mutating only GAG3 produced a strong mutant phenotype (Supplementary Fig. 3e-j).

Our findings imply a role for an as-yet-undefined Orion receptor on the surface of the glial cells. The glial receptor draper (drpr) seemed an obvious candidate ${ }^{13,28-30}$, although Drpr ligands unrelated to Orion have been identified ${ }^{31,32}$. The MB remodeling phenotypes in orion ${ }^{1}$ and $d r p r^{\Delta 5}$ are, however, different; only orion ${ }^{1}$ displayed unpruned axons and the $d r p r$ mutant phenotype does not persist throughout adulthood ${ }^{13}$ (Table I and Supplementary Fig. 1). Strikingly, using UAS-mGFP driven by $201 Y$-GAL4, instead of Fas2, where the labelling of $\alpha \beta$ axons often masks individual unpruned $\gamma$ axons, allowed us to observe occasionally unpruned axons in $d r p r^{\Delta 5}$ one-week-old post-eclosion brains (Table I and Supplementary Fig. 2) indicating a certain degree of undescribed axon persistence in the mutant background. In addition, our data indicate that Orion does not induce the Drpr signaling pathway

216 (Supplementary Fig. 10). This suggests that Drpr is not an, or at least not the sole, Orion 217 receptor.

218 We have uncovered a neuronally-secreted chemokine-like protein acting as a "find219 me/eat-me" signal involved in the neuron-glia crosstalk required for axon pruning during 220 developmental neuron remodeling. To the best of our knowledge, chemokine-like signaling in 221 insects was not described previously and furthermore our results point to an unexpected conservation of chemokine $\mathrm{CX}_{3} \mathrm{C}$ signaling in modulation of neural circuits.

\section{Methods}

\section{Drosophila stocks}

226 All crosses were performed using standard culture medium at $25^{\circ} \mathrm{C}$. Except where otherwise 227 stated, alleles have been described previously (http://flystocks.bio.indiana.edu). The following 
alleles were used. orion ${ }^{1}$, orion $^{\Delta A}$, orion $^{\Delta B}$ and orion $^{\Delta C}$ were generated in this study. drpr $^{45 r e c 8}$ was found to have an unlinked lethal mutation which was removed by standard mitotic recombination over a wild-type chromosome ${ }^{28,29}$. Animals bearing this version of $d r p r^{45}$ survive to adult stages and were used for this work. The following transgenes were used. UASorion-RNAi (VDRC stock 30843) and 2x UAS-drl-myc ${ }^{26}, 10 X-S t a t 92 E-G F P^{33}$. UAS-orion-A, UAS-orion-A-myc, UAS-orion-B, UAS-orion-B-myc, UAS-orion-B-Mut AX3C-myc, UASorion-B-Mut CX4C-myc, UAS-orion-B- $\triangle S P$-myc, UAS-orion-B-Mut GAG1-myc, UAS-orion-BMut GAG2-myc and UAS-orion-B-Mut GAG3-myc were generated in this study. We used three GAL4 lines: 201Y-GAL4 expressed in $\gamma$ MB neurons, alrm-GAL4 expressed exclusively in glial astrocytes ${ }^{34}$ and the pan-glial driver repo-GAL4 expressed in all glia ${ }^{35}$.

\section{Mutagenesis and screening}

240 EMS mutagenesis was carried out following the published procedure ${ }^{36}$. EMS treated $y w^{67 c 23}$ $s n^{3} F R T 19 A$ males were crossed to $F M 7 c / p h^{0} w$ females and stocks, coming from single $y w^{67 c 23}$ $s n^{3}$ FRT19A/ FM7c female crossed to FM7c males, were generated. Only viable $y w^{67 c 23} s n^{3}$ FRT19A chromosome bearing stocks were kept and $y w^{67 c 23} s n^{3}$ FRT19A; UAS-mCD8-GFP 201Y-GAL4/+ adult males from each stock were screened for MB neuronal remodeling defect with an epi-fluorescence microscope (Leica DM 6000).

\section{Mapping of orion}

248 To broadly map the location of the EMS-induced mutation on the X chromosome we used males from the stocks described in the X-chromosome duplication kit (Bloomington Stock Center) that we crossed with orion ${ }^{1}$; UAS-mCD8-GFP 201Y-GAL4 females. Dp(1; Y)BSC346 (stock 36487) completely rescued the orion $^{1} \gamma$ axon unpruned phenotype. This duplication is located at 6D3-6E2; 7D18 on the X chromosome. We then used smaller duplications covering this region. Thus, duplications Dp(1;3)DC496 (stock 33489) and Dp(1;3)DC183 (stock 32271) also rescued the orion $^{l}$ mutant phenotype. However duplication $\mathrm{Dp}(1 ; 3) \mathrm{DC} 184$ (stock 30312) did not rescue the mutant phenotype. Overlapping of duplications indicates that the EMS mutation was located between 7C9 and 7D2 which comprises $72 \mathrm{~kb}$. In addition, deficiency Def(1)C128 (stock 949, Bloomington Stock Center) which expand from 7D1 to 7D5-D6 complements orion $^{1}$ contrarily to deficiency Def(1)BSC622 (stock 25697, Bloomington Stock mutant MBs resembles a star constellation. 


\section{Whole-Genome Sequencing}

263 Gene mutation responsible for the unpruned $\gamma$ axon phenotype was precisely located through 264 the application of next generation sequencing. Genomic DNA was extracted from 30 adult 265 females (mutant and control) and directly sequenced on a HiSeq2000 next-generation 266 sequencing platform (Illumina). Bioinformatics analysis for read alignment and variant 267 investigation was carried out through the $72 \mathrm{~kb}$ selected by duplication mapping (see above) at 268 the University of Miami Miller School of Medicine, Center for Genome Technology.

\section{Signal peptide and transmembrane protein domain research}

271 For prediction of signal peptide sequences, we used the PrediSi website ${ }^{37}$ : 272 http://www.predisi.de.; for transmembrane domains, we used the TMHMM Server, v 2.0 $0^{38}$ : 273 http://www.cbs.dtu.dk/services/TMHMM/

\section{Orion and fractalkine alignments}

276 The sequence of the region common to both Orion isoforms containing the $\mathrm{CX}_{3} \mathrm{C}$ motifs and 277 the likely conserved $\mathrm{CX}_{3} \mathrm{C}$-downstream cysteines and those of the human, mouse and chicken 278 fractalkine were aligned using the AlignX plug-in in the VectorNTi software package 279 (InVitrogen) without permitting introduction of spaces or deletions.

\section{GAG binding site research}

282 Identification of GAG binding sites in proteins, in absence of structural data, is complicated by 283 the diversity of both GAG structure and GAG binding proteins. Previous work based on 284 heparin-binding protein sequence comparisons led to the proposition of two GAG binding consensus sequences, the XBBXBX and XBBBXXBX motifs (where B and X stand for basic and neutral/hydrophobic amino acids respectively). A number of closely related basic clusters, including XBBXBXBX were next experimentally identified ${ }^{39}$. Visual examination of the Orion-B sequences returned three such clusters (XBBXXBXXBXXBX: residues 242-254; XBBXBX: residues 416-421 and XBBXBXBX: residues 547-554, numbering includes the peptide signal see Fig. 2d), which are also present in Orion-A.

\section{CRISPR-Cas9 strategy}

293 All guide RNA sequences (sgRNA) were selected using the algorithm 294 targetfinder.flycrispr.neuro.brown.edu/ containing 20 nucleotides each (PAM excluded) and 295 are predicted to have zero off-targets. We selected three pairs of sgRNA. Each pair is targeting 
296 either the A specific region of orion, the B specific region of orion or the C common region of

297 the two isoforms. We used the following oligonucleotide sequences:

298 CRISPR-1 orion A fwd :

299 TATATAGGAAAGATATCCGGGTGAACTTCATTTGCGTTTTGATTTTCAGGTTTTAG

300 AGCTAGAAATAGCAAG

301 CRISPR-1 orion A rev :

302 ATTTTAACTTGCTATTTCTAGCTCTAAAACGCTGTTGGAGTAGATTGGTGGACGTT

303 AAATTGAAAATAGGTC

304 CRISPR-1 orion B fwd :

TATATAGGAAAGATATCCGGGTGAACTTCGTGAAATCTCAGCTGTATCGGTTTTA

GAGCTAGAAATAGCAAG

307 CRISPR-1 orion B rev : AAATTGAAAATAGGTC

310 CRISPR-1 orion commun region $\mathrm{C}$ fwd :

311 TATATAGGAAAGATATCCGGGTGAACTTCACCTGGTAAAGAATGCCAGAGTTTTA

312 GAGCTAGAAATAGCAAG

313 CRISPR-1 orion commun region $\mathrm{C}$ rev :

314 ATTTTAACTTGCTATTTCTAGCTCTAAAACCTTCGCGTCCAGGTGAGTCTGACGTT 315 AAATTGAAAATAGGTC

316 We introduced two sgRNA sequences into pCFD4 ${ }^{40}$, a gift from Simon Bullock (Addgene 317 plasmid \# 49411) by Gibson Assembly (New England Biolabs) following the detailed protocol 318 at crisprflydesign.org. For PCR amplification, we used the protocol described on that website. 319 Construct injection was performed by Bestgene (Chino Hills, CA) and all the transgenes were 320 inserted into the same attP site (VK00027 at 89E11). Transgenic males expressing the different 321 orion $\operatorname{sgRNAs}$ were crossed to $y$ nos-Cas $9 w^{*}$ females bearing an isogenized $\mathrm{X}$ chromosome. 322100 crosses were set up for each sgRNA pair, with up to 5 males containing both the sgRNAs 323 and nos-Cas9, and $5 F M 7 c / p h^{0} w$ females. From each cross, a single y nos-Cas $9 w^{*} / F M 7 c$ 324 female was crossed with $F M 7 c$ males to make a stock which was validated for the presence of 325 an indel by genomic PCR with primers flanking the anticipated deletion and the precise 326 endpoints of the deletion were determined by sequencing (Genewiz, France) using orion 327 specific primers. 


\section{Adult brain dissection, immunostaining and MARCM mosaic analysis}

Adult fly heads and thoraxes were fixed for $1 \mathrm{~h}$ in $3.7 \%$ formaldehyde in PBS and brains were dissected in PBS. For larval and pupal brains, brains were first dissected in PBS and then fixed for $15 \mathrm{~min}$ in $3.7 \%$ formaldehyde in PBS. They were then treated for immunostaining as previously described ${ }^{23,41}$. Antibodies, obtained from the Developmental Studies Hybridoma Bank, were used at the following dilutions: Mouse monoclonal anti-Fas2 (1D4) 1:10, mouse monoclonal anti-Draper (8A1), 1:400 and mouse monoclonal anti-Repo (8D1.2) 1:10. Mouse monoclonal primary antibody against EcR-B1 (AD4.4) was used at 1:5.000 ${ }^{42}$. Polyclonal mouse (Abcam, (9E10) ab32) and Rabbit (Cell Signaling 7D10) anti-Myc antibodies were used at 1: 1000 and 1: 500, respectively. Goat secondary antibodies conjugated to Cy3, Alexa 488 and Cy5 against mouse or rabbit IgG (Jackson Immunoresearch laboratory) were used at 1:300

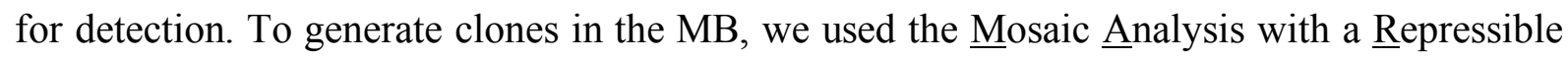
Cell Marker (MARCM) technique ${ }^{23}$. First instar larvae were heat-shocked at $37^{\circ} \mathrm{C}$ for $1 \mathrm{~h}$. Adult brains were fixed for $15 \mathrm{~min}$ in $3,7 \%$ formaldehyde in PBS before dissection and GFP visualization.

\section{Microscopy and image processing}

346 Images were acquired at room temperature using a Zeiss LSM 780 laser scanning confocal microscope (MRI Platform, Institute of Human Genetics, Montpellier, France) equipped with a 40x PLAN apochromatic 1.3 oil-immersion differential interference contrast objective lens. The immersion oil used was Immersol 518F. The acquisition software used was Zen 2011 (black edition). Contrast and relative intensities of the green (GFP), of the red (Cy3) and of the blue (Cy5 and Alexa 488) channels were processed with ImageJ and Fiji software. Settings were optimized for detection without saturating the signal. For each set of figures settings were constants. However, since the expression of the Orion-B-Myc- $\Delta$ SP protein is lower than the one of the Orion-B-Myc (as shown in the western blot and its quantitation Supplementary Fig. $3 \mathrm{i}-\mathrm{j}$ ), the levels of red were increased in this particular case in order to get similar levels than in Orion-B-Myc. We used the Imaris (Bitplane) software to generate a pseudo-3D structure of Orion-secreting $\gamma$ axons (Imaris surface tool). We created two 3D surfaces, from regular confocal images, defining the axonal domain (green) and the Orion secretion domain (red).

\section{Quantitation of immunolabelling}

361 To quantify unpruned $\gamma$ axons we stablished three categories of phenotypes: "none", when no 362 unpruned axons are observed, "weak", when few unpruned individual axons or thin axon 
bundles are observed in the dorsal lobe and "strong", when $>50 \%$ of the axons are unpruned. In this last category, the percentage of unpruned axons is estimated by the width of the corresponding medial bundle compared with the width of the medial pruned axon bundle ${ }^{41}$. For debris quantification we established five categories : none, scattered dots, mild, intermediary and strong based on the location and size of the debris clusters ${ }^{11}$. In $\geq$ one-week-old adults, "none" means absence of debris. In $\leq$ two-hours-old adults "scattered dots" means some individual debris can be observed. We considered "mild", if debris clusters (clusters $>5 \mu \mathrm{m}^{2}$ ) appear only at one location, "intermediary", at two locations and "strong" at three locations of the MB. Three debris locations were considered: the tip of the vertical lobe, the tip of the medial lobe and around the heel (bifurcation site of $\gamma$ axons into dorsal and medial). For EcR-B1 signal quantitation, we performed 5 measurements for each picture (Intensity 1,.., 5) in the nuclei of GFP positive cell bodies and the same number of measurements in background using confocal single slices. The mean of these background measurements is called mean background. We then subtracted intensities of mean background from each intensity value (Intensity $1, \ldots, 5$ minus mean background) to obtain normalized intensity values. Finally, we compared normalized intensity values between two genetic conditions. We proceeded similarly for Draper and STAT-GFP signal quantitation, but staining was quantified in the astrocyte cytoplasm located in the immediate vicinity of the $\gamma$ dorsal lobe. Quantitation of intensity was performed using Image J software.

To quantify the Myc signal in the $\gamma$ vertical lobe, we traced a $60 \mu \mathrm{m}$ line on the Cy3 red Z-stack and used the Plot Profile function of ImageJ to create a plot of intensity values across the line. The top of the line $(0 \mu \mathrm{m})$ was located at the tip of the $\gamma$ vertical lobe and the bottom of the line $(60 \mu \mathrm{m})$ at the branching point of the two $\gamma$ lobes. Only images containing an astrocyte sitting at the top of the $\gamma$ vertical lobe were used to quantify Myc expression levels in orion- $B$ and orion- $B-\triangle S P$ expressing MBs.

To quantify the number of astrocytes around the $\gamma$ lobes, we counted the number of glial nuclei, as labelled with anti-Repo antibody, contained in GFP-positive astrocyte cytoplasm labelled with $U A S-m C D 8-G F P$ driven by alrm-GAL4. We only counted nuclei contained within a circle of $70 \mu \mathrm{m}$ of diameter centered in the middle of the vertical $\gamma$ lobe tips.

\section{UAS constructs}

394 The orion- $A$ cDNA inserted in the pOT2 plasmid (clone LD24308) was obtained from Berkeley 395 Drosophila Genome Project (BDGP). Initial Orion-B $c D N A$ as well as the Orion-B $c D N A s$ 
containing mutations at the $\mathrm{CX}_{3} \mathrm{C}$ and the $\mathrm{GAG}$ sites or lacking the signal peptide were produced at GenScript (Piscataway, NJ) in the pcDNA3.1-C-(k)DYK vector. The Orion-B $c D N A s$ contained the following mutations:

To remove the signal peptide, we removed sequence: GCGCCGCCTTTCGGATTATTAGCTGCT GTTGTTGCTGTTCTTGTCACGCTTGTGATTTGTGGAAATA located after the first ATG. At the $\mathbf{C X}_{3} \mathrm{C}$ site: $\mathrm{In} \mathbf{A X}_{3} \mathrm{C}$ we exchanged TGC to GCC. In CX4C, we added an additional Ala (GCC) to get $\mathrm{CAX}_{3} \mathrm{C}$.

To mutate the putative GAG binding sites we exchanged Lys and Arg by Ala at the corresponding sites: AAGAGGACGGAACGCACACTAAAAATACTCAAG; AAGCGCAACCGA and CGCAGGGAGAAACTGCGT to GCCGCCACGGAAGCCACACTAGCCATACTCAAG, GAG2 and GAG3.

The different constructs were amplified by PCR using forward primers containing sequences CACCaaaacATG (where ATG encodes the first Methionine) followed by the specific orion- $A$ or orion- $B C D N A$ sequences and including or not nucleotides corresponding to the STOP codon at the reverse primers resulting in transgenes without and with a MYC-tag respectively.

414 To amplify orion-A we used as forward primers $(\mathrm{F})$ :

415 F: CACCAAAACATGAGATTTATAAATTGGGTACTTCCCCT

416 To amplify orion-B we used:

417 F: CACCAAAACATGGCGCCGCCTTTCGGATTATTA

418 For both we used the same reverse oligonucleotide $(\mathrm{R})$ :

419 R containing stop: TTAGAATCTATTCTTTGGCACCTGAACGT

420 R without stop: GAATCTATTCTTTGGCACCTGAACGT

Amplified cDNA was processed for pENTR/D-TOPO cloning (ThermoFisher Scientific, K240020) and constructs were subsequently sequenced (Genewiz, France). We used the Gateway LR clonase enzyme mix (ThermoFisher Scientific, 11791019) to recombine the

424 inserts into the destination UAS vector pJFRC81-GW-2xMyc (L. G. F., unpublished) which was generated from pJFRC81-10XUAS-IVS-Syn21-GFP-p10 (Addgene plasmid 36462 deposited by G. Rubin ${ }^{43}$ ) by replacing the GFP ORF with a Gateway cassette adding on a Cterminal 2x Myc tag. orion- $A$, orion- $B$ and Myc-tagged constructs (orion A, orion B, and orion- 
system were performed at $25^{\circ} \mathrm{C}$ except for $U A S$-orion- $A$ and $201 Y$-GAL4 which were performed at $18^{\circ} \mathrm{C}$.

\section{Western analysis}

434 Five larval heads were homogenized in an Eppendorf tube containing $20 \mu 1$ of $3 \mathrm{X}$ sample buffer (2\% SDS, 0.125 M Tris- $\mathrm{HCl}$ pH 6.9, 5\% $\beta$-mercaptoethanol, 20\% glycerol, bromophenol blue) and proteins were separated by SDS-PAGE. After electrotransfer to nitrocellulose, the blot was blocked in PBS, 0.5\% Tween-20, 5\% milk. The Orion-Myc and Tubulin proteins were detected using a mouse anti-Myc antibody (clone 9E10, AbCam) and an anti-Tubulin antibody (Sigma, T5168) at $1 / 1000$ and $1 / 10.000$ respectively in PBS, 0.5\% Tween-20, 5\% milk and revealed using anti-mouse Ig horseradish peroxidase (1:10.000) and an ECL kit (Amersham). Band intensities were normalized to the corresponding tubulin band intensity using the ImageJ software.

\section{Statistics}

445 Comparison between two groups expressing a qualitative variable was analyzed for statistical 446 significance using the Fisher's exact test for a $2 \times 3$ contingency table 447 (https://www.danielsoper.com/statcalc/calculator.aspx?id=58). Comparison of two groups 448 expressing a quantitative variable was analyzed using the two-tailed non-parametric Mann-

449 Whitney $U$ test (https://www.socscistatistics.com/tests/mannwhitney/Default2.aspx). Values of $450 \mathrm{p}<0.05$ were considered to be significant. Graphs were performed using the GraphPad Prism 451 software (version 8.1.1).

\section{References}

4541 Luo, L. \& O'Leary, D. D. Axon retraction and degeneration in development and disease. $455 \quad$ Annu Rev Neurosci 28, 127-156 (2005).

4562 Neukomm, L. J. \& Freeman, M. R. Diverse cellular and molecular modes of axon 457 degeneration. Trends Cell Biol 24, 515-523, doi:10.1016/j.tcb.2014.04.003 (2014).

4583 Schuldiner, O. \& Yaron, A. Mechanisms of developmental neurite pruning. Cell Mol Life 459 Sci 72, 101-119, doi:10.1007/s00018-014-1729-6 (2015).

4604 Neniskyte, U. \& Gross, C. T. Errant gardeners: glial-cell-dependent synaptic pruning 461 and neurodevelopmental disorders. Nat Rev Neurosci 18, 658-670, 462 doi:10.1038/nrn.2017.110 (2017). evidence for local degeneration and requirement of the ubiquitin-proteasome system. Neuron 38, 871-885 (2003). 
4666 Yu, F. \& Schuldiner, O. Axon and dendrite pruning in Drosophila. Curr Opin Neurobiol 27, 192-198, doi:10.1016/j.conb.2014.04.005 (2014).

4687 Boulanger, A. \& Dura, J. M. Nuclear receptors and Drosophila neuronal remodeling. Biochim Biophys Acta 1849, 187-195, doi:10.1016/j.bbagrm.2014.05.024 (2015).

8 Yaniv, S. P. \& Schuldiner, O. A fly's view of neuronal remodeling. Wiley Interdiscip Rev Dev Biol 5, 618-635, doi:10.1002/wdev.241 (2016).

9 Awasaki, T. \& Ito, K. Engulfing action of glial cells is required for programmed axon pruning during Drosophila metamorphosis. Curr Biol 14, 668-677, doi:10.1016/j.cub.2004.04.001

47610 Watts, R. J., Schuldiner, O., Perrino, J., Larsen, C. \& Luo, L. Glia engulf degenerating

11 Tasdemir-Yilmaz, O. E. \& Freeman, M. R. Astrocytes engage unique molecular programs to engulf pruned neuronal debris from distinct subsets of neurons. Genes Dev 28, 20-33, doi:10.1101/gad.229518.113 (2014).

12 Hakim, Y., Yaniv, S. P. \& Schuldiner, O. Astrocytes play a key role in Drosophila mushroom body axon pruning. PLoS One 9, e86178, doi:10.1371/journal.pone.0086178 (2014).

13 Awasaki, T. et al. Essential role of the apoptotic cell engulfment genes draper and ced6 in programmed axon pruning during Drosophila metamorphosis. Neuron 50, 855-867 (2006).

14 Lee, T., Marticke, S., Sung, C., Robinow, S. \& Luo, L. Cell-autonomous requirement of the USP/EcR-B ecdysone receptor for mushroom body neuronal remodeling in Drosophila. Neuron 28, 807-818 (2000).

15 Zheng, X. et al. TGF-beta signaling activates steroid hormone receptor expression during neuronal remodeling in the Drosophila brain. Cell 112, 303-315 (2003).

16 Paolicelli, R. C., Bisht, K. \& Tremblay, M. E. Fractalkine regulation of microglial physiology and consequences on the brain and behavior. Front Cell Neurosci 8, 129, doi:10.3389/fncel.2014.00129 (2014).

17 Arnoux, I. \& Audinat, E. Fractalkine Signaling and Microglia Functions in the Developing Brain. Neural Plast 2015, 689404, doi:10.1155/2015/689404 (2015).

18 Werneburg, S., Feinberg, P. A., Johnson, K. M. \& Schafer, D. P. A microglia-cytokine axis to modulate synaptic connectivity and function. Curr Opin Neurobiol 47, 138-145, doi:10.1016/j.conb.2017.10.002 (2017). the cross-talk between neuron and glia in neurological diseases. Brain Res Bull 146, 12 21, doi:10.1016/j.brainresbull.2018.11.017 (2019). Wilton, D. K., Dissing-Olesen, L. \& Stevens, B. Neuron-Glia Signaling in Synapse Elimination. Annu Rev Neurosci 42, 107-127, doi:10.1146/annurev-neuro-070918050306 (2019). and fractalkine signaling. Nat Neurosci 22, 1075-1088, doi:10.1038/s41593-019-0419y (2019). function in neuronal morphogenesis. Neuron 22, 451-461 (1999). 
24 Alyagor, I. et al. Combining Developmental and Perturbation-Seq Uncovers Transcriptional Modules Orchestrating Neuronal Remodeling. Dev Cell 47, 38-52 e36, doi:10.1016/j.devcel.2018.09.013 (2018).

25 Hoopfer, E. D., Penton, A., Watts, R. J. \& Luo, L. Genomic analysis of Drosophila neuronal remodeling: a role for the RNA-binding protein Boule as a negative regulator of axon pruning. J Neurosci 28, 6092-6103 (2008).

26 Reynaud, E. et al. Guidance of Drosophila Mushroom Body Axons Depends upon DRLWnt Receptor Cleavage in the Brain Dorsomedial Lineage Precursors. Cell Rep 11, 1293-1304, doi:10.1016/j.celrep.2015.04.035 (2015).

522

27 Monneau, Y., Arenzana-Seisdedos, F. \& Lortat-Jacob, H. The sweet spot: how GAGs

help chemokines guide migrating cells. J Leukoc Biol 99, 935-953, doi:10.1189/jlb.3MR0915-440R (2016). $\mathrm{Gcm}$ target genes regulating glial development, diversification, and function. Neuron 38, 567-580, doi:10.1016/s0896-6273(03)00289-7 (2003).

29 MacDonald, J. M. et al. The Drosophila cell corpse engulfment receptor Draper mediates glial clearance of severed axons. Neuron 50, 869-881, doi:10.1016/j.neuron.2006.04.028 (2006).

30 Musashe, D. T., Purice, M. D., Speese, S. D., Doherty, J. \& Logan, M. A. Insulin-like Signaling Promotes Glial Phagocytic Clearance of Degenerating Axons through Regulation of Draper. Cell Rep 16, 1838-1850, doi:10.1016/j.celrep.2016.07.022 (2016).

32 Lin, L. et al. Complement-Related Regulates Autophagy in Neighboring Cells. Cell 170, 158-171 e158, doi:10.1016/j.cell.2017.06.018 (2017).

31 Kuraishi, T. et al. Pretaporter, a Drosophila protein serving as a ligand for Draper in the phagocytosis of apoptotic cells. EMBO J 28, 3868-3878, doi:10.1038/emboj.2009.343 (2009).

33 Bach, E. A. et al. GFP reporters detect the activation of the Drosophila JAK/STAT pathway in vivo. Gene Expr Patterns 7, 323-331, doi:10.1016/j.modgep.2006.08.003 (2007).

34 Doherty, J., Logan, M. A., Tasdemir, O. E. \& Freeman, M. R. Ensheathing glia function as phagocytes in the adult Drosophila brain. J Neurosci 29, 4768-4781, doi:29/15/4768 [pii]

10.1523/JNEUROSCI.5951-08.2009 (2009).

35 Sepp, K. J., Schulte, J. \& Auld, V. J. Developmental dynamics of peripheral glia in Drosophila melanogaster. Glia 30, 122-133 (2000).

36 Lewis, E. B. \& Bacher, F. Method of feeding ethyl methane sulfonate (EMS) to Drosophila males. Dros. Inf. Serv., 193 (1968).

37 Hiller, K., Grote, A., Scheer, M., Munch, R. \& Jahn, D. PrediSi: prediction of signal peptides and their cleavage positions. Nucleic Acids Res 32, W375-379, doi:10.1093/nar/gkh378 (2004).

38 Krogh, A., Larsson, B., von Heijne, G. \& Sonnhammer, E. L. Predicting transmembrane protein topology with a hidden Markov model: application to complete genomes. $J$ Mol Biol 305, 567-580, doi:10.1006/jmbi.2000.4315 (2001).

39 Vives, R. R. et al. A novel strategy for defining critical amino acid residues involved in protein/glycosaminoglycan interactions. J Biol Chem 279, 54327-54333, doi:10.1074/jbc.M409760200 (2004). 
40 Port, F., Chen, H. M., Lee, T. \& Bullock, S. L. Optimized CRISPR/Cas tools for efficient

\section{Acknowledgements}

We thank Amélie Babled, Pascal Carme and Dana Bis-Brewer for help in the EMS mutagenesis, MB developmental studies and WGS analysis respectively, Oren Schuldiner for discussions about the Orion expression and function. We thank Marc Freeman for alrm-GAL4 stock, Baeg Gyeong Hun for 10X-STAT92E-GFP stock, the Bloomington Drosophila Stock Center and VDRC for fly stocks, the Drosophila facility, BioCampus Montpellier, CNRS, supported by the French National Research Agency (ANR-10-INBS-04) for help in confocal and image analysis and processing. We acknowledge BDGP, BestGene, GenScript and Genewiz for cDNA clone, transgene service, gene synthesis and DNA sequencing, respectively. The 1D4 anti-Fasciclin II hybridoma and the 8D12 anti-Repo monoclonal antibody developed by Corey Goodman and the 8A1 anti-Draper monoclonal antibody developed by Mary Logan were obtained from the Developmental Studies Hybridoma Bank, created by the NICHD of the NIH and maintained at The University of Iowa, Department of Biology, Iowa City, IA 52242.

597 Funding: C.T. was supported by grants from the INSB at the CNRS and from the Fondation 
599 National de la Recherche Scientifique, the Association pour la Recherche sur le Cancer (grants 600 SFI20121205950 and PJA 20151203422) and the Fondation pour la Recherche Médicale 601 (Programme "EQUIPES FRM2016" project DEQ20160334870).

602

\section{Author contributions}

604 A.B. and J.-M.D. designed the project; A.B., C.T. and J.-M.D. performed the experiments; 605 A.B., S.Z., L.G.F., H.L.-J. and J.-M.D. analyzed the data; A.B. and J.-M.D. wrote the original 606 draft of the manuscript; A.B., L.G.F., H.L.-J. and J.-M.D. reviewed and edited the manuscript. 607

\section{Competing interests}

609 The authors declare no competing interests.

610

\section{Additional information}

612 Supplementary information: Supplementary Fig. 1 to 10 and Supplementary list of fly strains 613

614 
Fig. 1
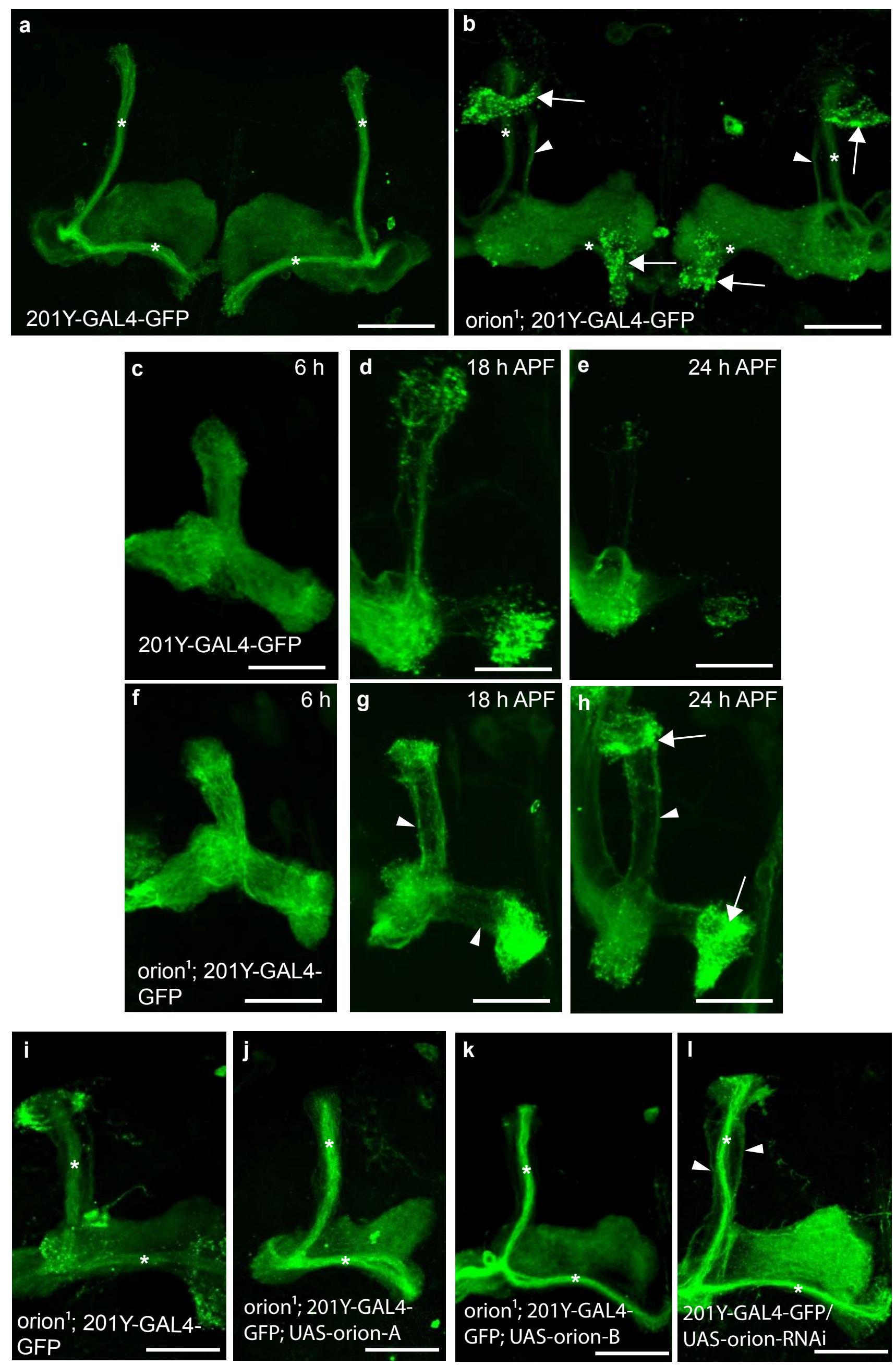
616 Fig. 1. The orion gene is necessary for MB remodeling. a-l, $\gamma$ neurons are visualized by the 617 expression of $201 Y$-GAL4 driven UAS-mCD8-GFP (green). In adults, this GAL4 line also 618 labels the $\alpha \beta$-core axons shown here by asterisks. a, b, Adult $\gamma$ axons in control (a) and orion ${ }^{1}$ 619 (b). Note the presence of unpruned $\gamma$ axon bundles (arrowhead) and the high amount of 620 uncleared axonal debris (arrows) in orion $^{1}$ compared to wild-type ( $\mathrm{n} \geq 100 \mathrm{MBs}$ for control and 621 orion $^{l}$. See quantitation in Table I and Supplementary Fig. 2). c-h, $\gamma$ axon development in wild622 type (c-e) and orion $^{l}$ (f-h) at $6 \mathrm{~h}, 18 \mathrm{~h}$ and $24 \mathrm{~h} \mathrm{APF}$ as indicated. Unpruned axons (arrowhead)

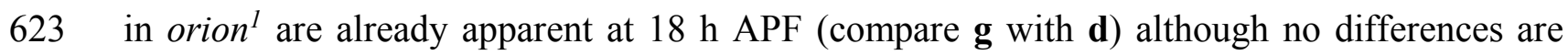
624 detected at $6 \mathrm{~h} \mathrm{APF} \mathrm{(c} \mathrm{and} \mathrm{f).} \mathrm{Note} \mathrm{the} \mathrm{presence} \mathrm{of} \mathrm{unpruned} \gamma$ axons (arrowhead) and debris 625 (arrow) in orion $^{1}$ at $24 \mathrm{~h} \mathrm{APF} \mathrm{(} \mathrm{n} \geq 40 \mathrm{MBs}$ for each developmental stage). i-k, The adult orion ${ }^{1}$ 626 phenotype (i) is completely rescued by expression in MBs of $U A S$-orion- $A$ ( $\mathrm{n}=89 \mathrm{MBs})(\mathbf{j})$ or $627 U A S$-orion-B $(\mathrm{n}=387 \mathrm{MBs})(\mathbf{k}) . \mathbf{l}, U A S$-orion- $R N A i$ expression in MBs results in unpruned $\gamma$ 628 axon phenotypes (arrowheads) $(\mathrm{n}=20 \mathrm{MBs})$. Scale bars represent $40 \mu \mathrm{m}$. All the images are 629 composite confocal images. Genotypes are listed in Supplementary list of fly strains. 
Table I

\section{Presence of unpruned axons in $\geq$ one-week-old adults}

a

\begin{tabular}{|l|r|r|r|r|}
\hline & MB & None & Weak & Strong \\
\hline WT & 25 & 25 & 0 & 0 \\
\hline Hr39 & 22 & 0 & 0 & 22 \\
\hline orion $\Delta C$ & 22 & 0 & 22 & 0 \\
\hline orion1 & 20 & 0 & 20 & 0 \\
\hline orionRNAi & 34 & 0 & 34 & 0 \\
\hline drpr $\Delta 5$ & 22 & 20 & 2 & 0 \\
\hline
\end{tabular}

\section{Presence of axon debris in $\geq$ one-week-old adults}

b

\begin{tabular}{|l|r|r|r|r|r|}
\hline & MB & None & Mild & Intermediary & Strong \\
\hline WT & 25 & 25 & 0 & 0 & 0 \\
\hline Hr39 & 22 & 22 & 0 & 0 & 0 \\
\hline orion $\Delta$ C & 22 & 0 & 0 & 0 & 22 \\
\hline orion1 & 20 & 0 & 0 & 0 & 20 \\
\hline orionRNAi & 34 & 34 & 0 & 0 & 0 \\
\hline drpr $\triangle 5$ & 22 & 16 & 2 & 2 & 2 \\
\hline
\end{tabular}

Presence of axon debris in $\leq 2 \mathrm{~h}$-old adults

C

\begin{tabular}{|l|r|r|r|r|r|}
\hline & MB & Scattered dots & Mild & Intermediary & Strong \\
\hline WT & 10 & 10 & 0 & 0 & 0 \\
\hline orion $\Delta C$ & 12 & 0 & 0 & 0 & 12 \\
\hline drpr $\Delta 5$ & 73 & 40 & 11 & 4 & 18 \\
\hline
\end{tabular}


631 Table I. Unpruned axon and axon debris quantitation. Genotypes are indicated on the left.

632 "MB" indicates the number of mushroom bodies observed for each genotype. Unpruned axons

633 were ranked in three categories: "None" indicates the absence of unpruned $\gamma$ axons, "Weak"

634 and "Strong" refer to different levels of the mutant pruning phenotype. Axon debris were ranked

635 in five categories: "None" indicates the absence of debris, "Scattered dots" means that some

636 individual debris can be observed. "Mild", "Intermediate" and "Strong" refer to different levels

637 of debris (see Supplementary Fig. 2 and Methods). Full genotypes are listed in Supplementary

638 list of fly strains.

639 
Fig. 2

a

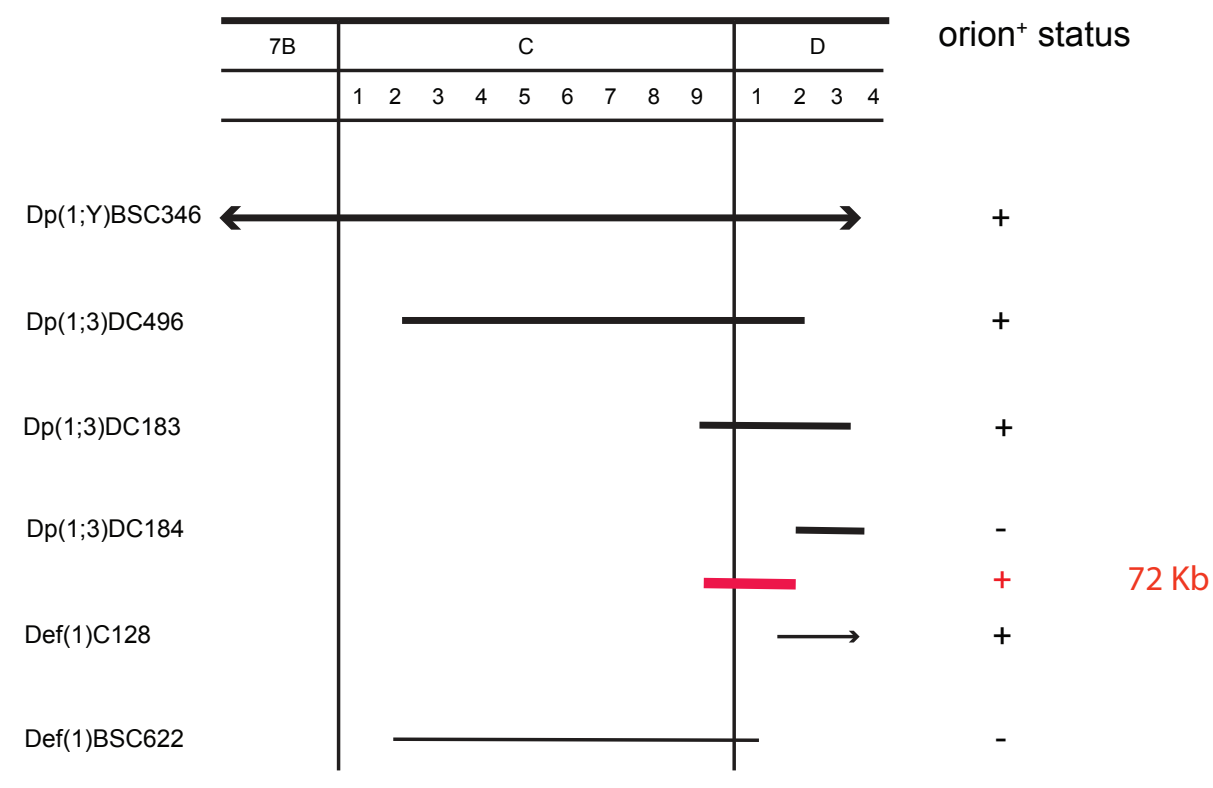

b
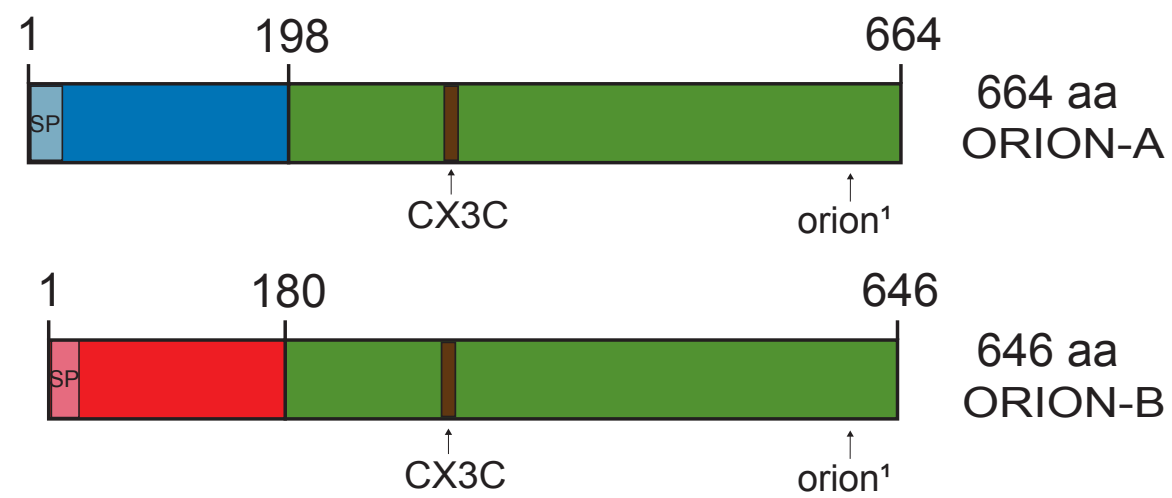

646 aa ORION-B

C

intramolecular disulfide bonds

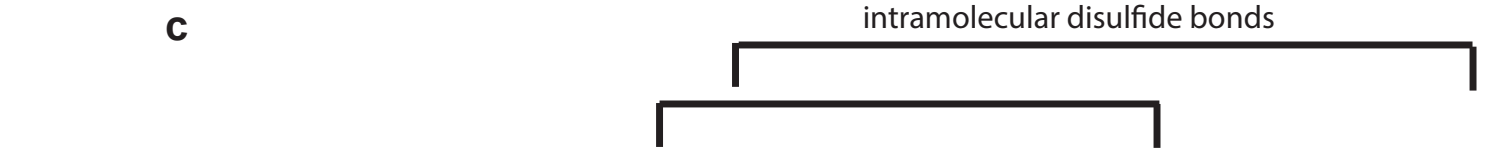

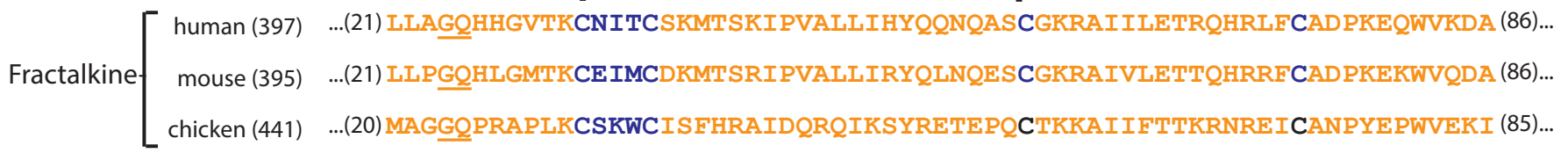

Orion $\begin{array}{ll}(\text { A 664) ...(307) ENEVDLNKEETCRETCDFYQSTRSEGCFKDLYCSRQPKCSGRLYNCRFVDSDMWVCPSPQNSTRR } \\ \text { (B 646) ...(289) }\end{array}$ CXXXC

d

\section{ORION B}

MAPPFGLLAAVVAVLVTLVICGNTKSASSEGWMHPSTPQMEVDALRTEYIALERALWNYLA KTANSQNNKETQIRKVYDSHRDFDAKPQMRRTFEEHRYKILNHYEWSLLERDLIYISKLYD AYKDTFVKQSNSVELDELAVLNLAGAILRNDNTASMPRILQEIERVMVSQTLYYRAMI * VSTDQICNTMQSAQQF IYSLYADIALTELKAYTMMEF SWMMLRVYGKGNYTQEAE IMRSEY

GAG1 EKRTERTLKILKDVMLRADRIVYRCDPTKHVRGVTYDEVTRLLQGYIENEVDLNKEETCRE TCDFYQSTRSEGCFKDLYCSRQPKCSGRLYNCRFVDSDMWVCPSPQNSTRRYEF IEYENGR VLGQRGKCTRGTTKVDSWWRYLLWHCSYCFCLCDEEGLKSDRFFNLRDTIADVKRNRVVTG $\mathrm{CX} 3 \mathrm{C}$ LRFVKQNRIFHLQIQEGELLPRGIVNQSTLEWKPVEKYNVFDRHVKNGVDYHKLSYEKRTI DLDDVDTDDNSFVVTGVRFRVVGTHLNLEAYYSEFDFRTGQLIRPEYNSYWKSNDNTDVSG

GAG3 ARREKLRLSNADVSTRTIAHSIPLSRHNQYIDFTNTGLDKDAAQSTVPFMDIQDVVSNPPV PLAGIGIYYKGRNGYGGFLGPKIITYDFMPHVQVPKNRF 
640 Fig. 2. The orion gene encodes for a $\mathbf{C X} \mathbf{X}_{3} \mathbf{C}$ motif-containing protein. a, Complementation 641 map of orion with the tested duplications and deficiencies in the 7B-7D region. Duplications 642 are drawn with a heavy line and deficiencies with a light line. If orion $^{+}$is present on the 643 chromosome carrying a duplication or deficiency it is indicated in the status column with a "+"; 644 and if it is not present it is marked "_.". The red line indicates the location of the $72 \mathrm{~kb}$ to which 645 orion is mapped based on the complementation results. b, Linear representation of the 646 polypeptide chain of the two Orion isoforms. Green represents the common region of the two 647 Orion proteins, blue is the specific N-terminal region of Orion-A and red the specific $\mathrm{N}$-terminal 648 region of Orion-B. The signal peptide of Orion-A and Orion-B (SP) are colored in light blue or 649 light red, respectively. The $\mathrm{CX}_{3} \mathrm{C}$ chemokine-motif as well as the location of the orion ${ }^{1}$ 650 mutation present in the common region of Orion-A and Orion-B are indicated. c, Amino acid 651 sequence lineups of human, mouse and chicken fractalkines with the common $\mathrm{CX}_{3} \mathrm{C}$-bearing 652 motif of the Drosophila Orion proteins is shown. The number in parenthesis after the species' 653 names indicate the total length of the protein. The underlined sequences in the fractalkine 654 sequences indicate the junctions at which their signal peptides are cleaved. The numbers at the 655 beginning and end of the sequence indicate the protein regions in the lineup. The $\mathrm{CX}_{3} \mathrm{C}$ 656 (CXXXC) and conserved downstream cysteines in the fractalkine species are indicated in blue. 657 Fractalkine intramolecular disulfide bonds between conserved cysteines ${ }^{44}$ are specified with 658 brackets. The $\mathrm{CX}_{3} \mathrm{C}$ motif in the Orions and the downstream cysteines are indicated in green. 659 The Orion downstream cysteines are offset by one and two amino acids, respectively, from 660 those in fractalkine relative to the $\mathrm{CX} 3 \mathrm{C}$ motif cysteines. The Orions differ from fractalkine 661 by the inclusion of considerable extensions upstream to the $\mathrm{CX}_{3} \mathrm{C}$ motif while the fractalkine $662 \mathrm{CX}_{3} \mathrm{C}$ motifs lie within 10 amino acids of the mature signal peptide-cleaved proteins. $\mathbf{d}$, Orion663 B amino-acid sequence where the signal peptide is in bold, the three putative GAG binding 664 sites (GAG1, GAG2, GAG3) are highlighted in yellow, the basic residues involved in GAG 665 binding $(\mathrm{R}=\operatorname{Arg}$ and $\mathrm{K}=\mathrm{Lys})$ are in red and the $\mathrm{CX}_{3} \mathrm{C}$ site is in brown. An asterisk is located 666 at the end of the Orion-B specific amino-acid sequence/beginning of the common region. The 667 glycine (GGC) which is mutated to an aspartic acid (GAC) in orion $^{l}$ is indicated by an arrow. 
Fig. 3

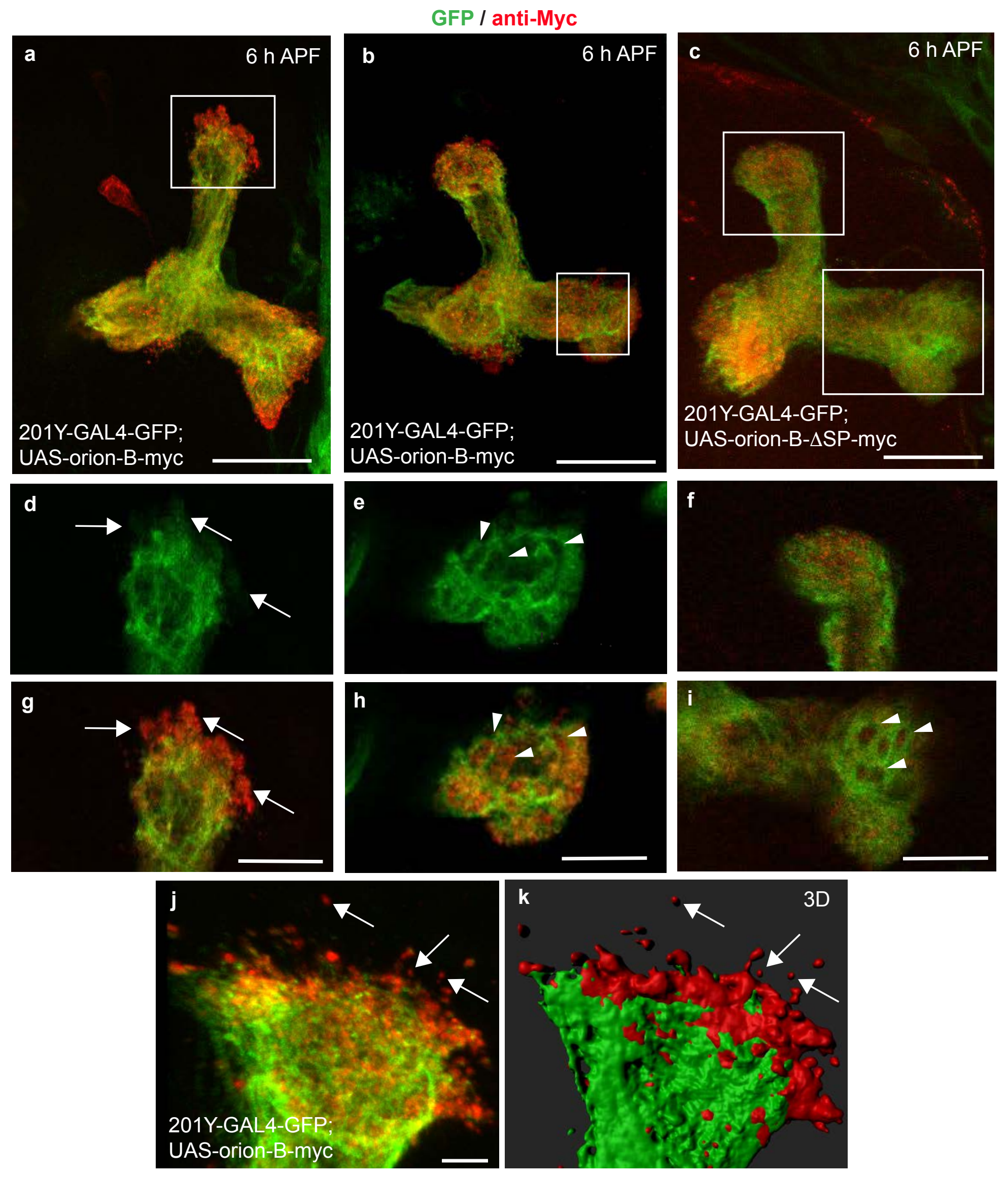


669 Fig. 3. Orion is secreted by MB $\gamma$ axons. a-k $6 \mathrm{~h}$ APF $\gamma$ axons are visualized by the expression 670 of $201 Y$-GAL4-driven UAS-mCD8-GFP (green). $\mathbf{a}, \mathbf{b}, \mathbf{j}, \mathbf{k}, \boldsymbol{\gamma}$ axons expressing the wild-type 671 Orion-B-Myc protein (red) ( $\mathrm{n} \geq 10 \mathrm{MBs}$ ). c, $\gamma$ axons expressing the Orion-B-Myc protein 672 lacking the signal peptide $(\Delta \mathrm{SP})(\mathrm{n}=9 \mathrm{MBs})$. a-c are confocal Z-projections and $\mathbf{j}$ is a unique 673 confocal plane. $\mathbf{d}, \mathbf{g}$, higher magnification images of the region indicated by rectangle in a 674 showing a representative unique confocal plane. Note the presence of Myc-labelled Orion-B 675 outside of the $\gamma$ axon bundle (arrows). $\mathbf{e}, \mathbf{h}$, higher magnification images of the region indicated 676 by rectangle in b showing a representative unique confocal plane. Note the presence of Myc677 labelled Orion-B inside of the hole-like structures present in the $\gamma$ axon bundle (arrowheads). $\mathbf{f}$, $678 \mathrm{i}$, higher magnification images of the vertical and medial $\gamma$ lobes, respectively (rectangles in $\mathbf{c}$ ). 679 Orion-B- $\Delta$ SP-Myc is not observed neither outside of the $\gamma$ axons (f) nor in the hole-like 680 structures (arrowheads in i). $\mathbf{j}, \mathbf{k}$ Presence of Myc-labelled Orion-B secreted proteins not 681 associated with GFP-labelled axon membranes can be observed outside of the $\gamma$ axon bundle 682 (arrows). $\mathbf{k}$, Three-dimensional surface-rendering (3D) of the confocal image. $\mathbf{j}$, reveals close 683 apposition of GFP-labelled axons and Myc-labelled Orion and reveals Orion is present as small 684 extracellular globules. Scale bars represent $40 \mu \mathrm{m}$ in a-c, $20 \mu \mathrm{m}$ in d-i and $5 \mu \mathrm{m}$ in j, k. Full 685 genotypes are listed in Supplementary list of fly strains. 
bioRxiv preprint doi: https://doi. org/10.1101/2020.11.23.394049; this version posted November 23, 2020. The copyright holder for this

preprint (which was not certified by peer review) is the author/funder. All rights reserved. No reuse allowed without permission.

anti-Fas2 / GFP
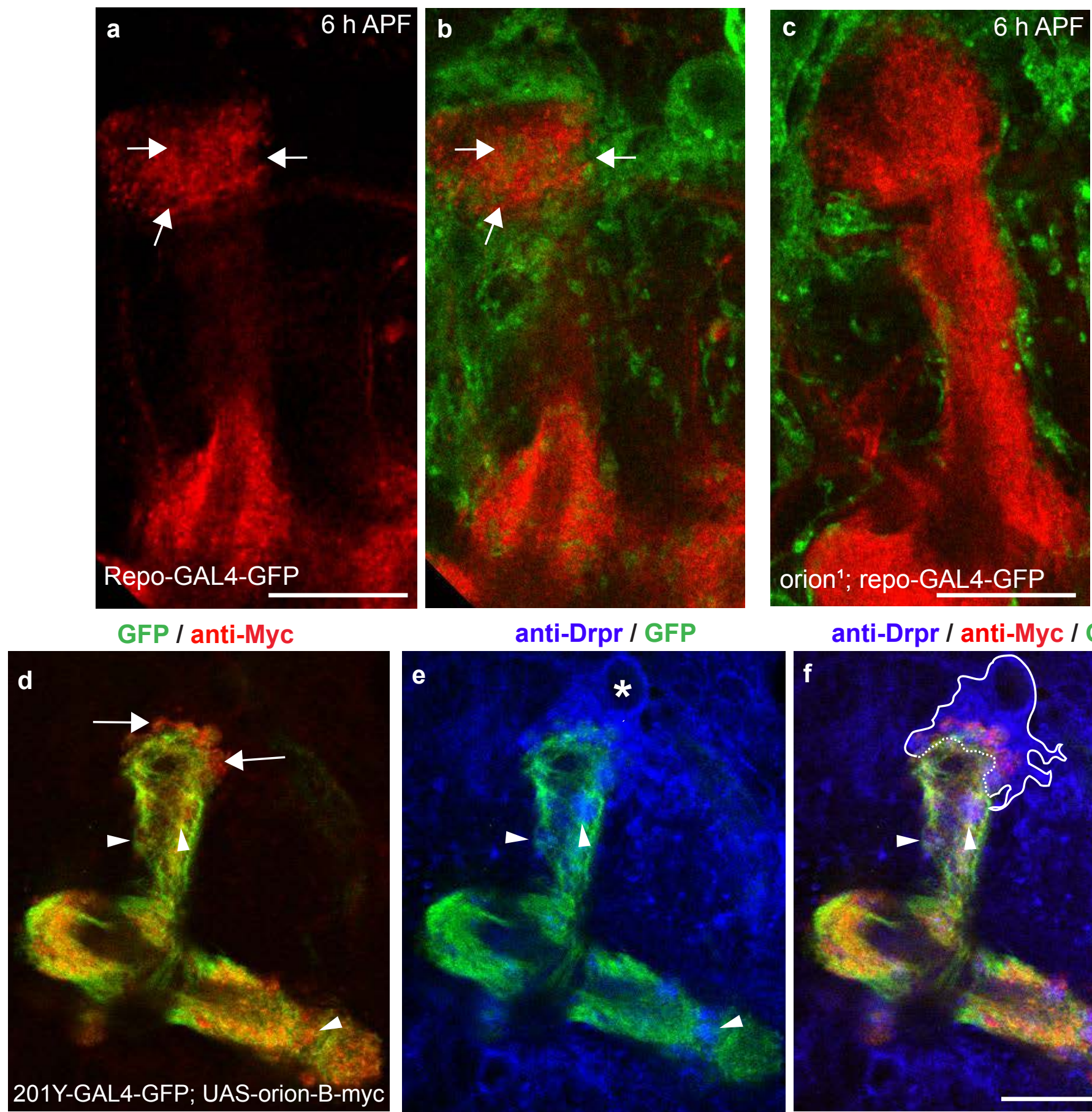

anti-Drpr / GFP

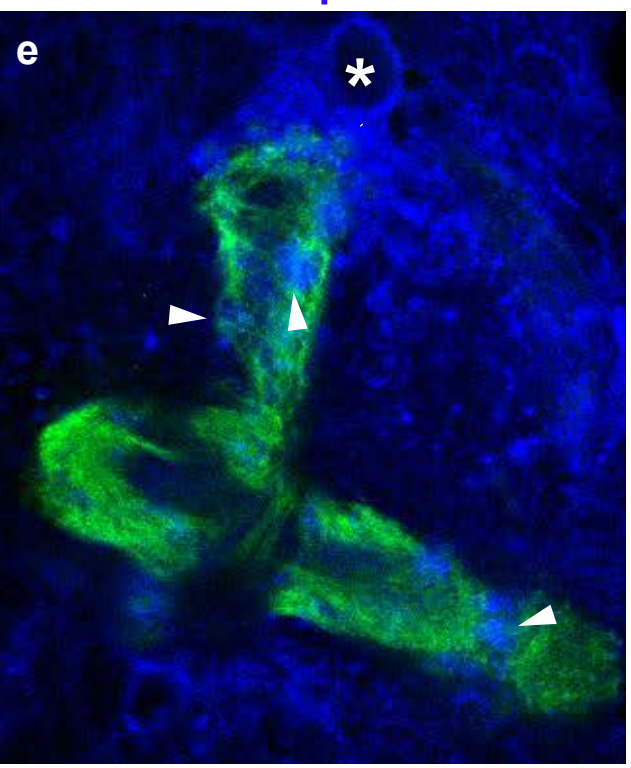

anti-Drpr / anti-Myc / GFP

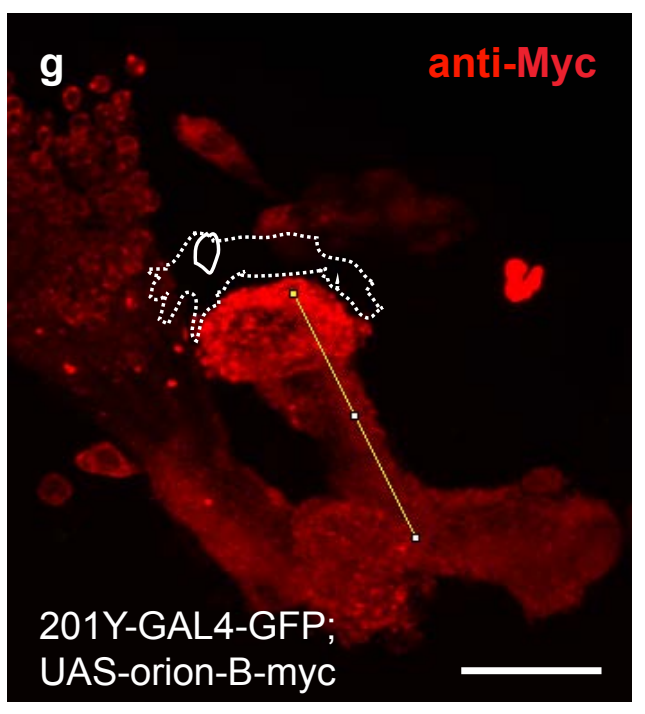

h
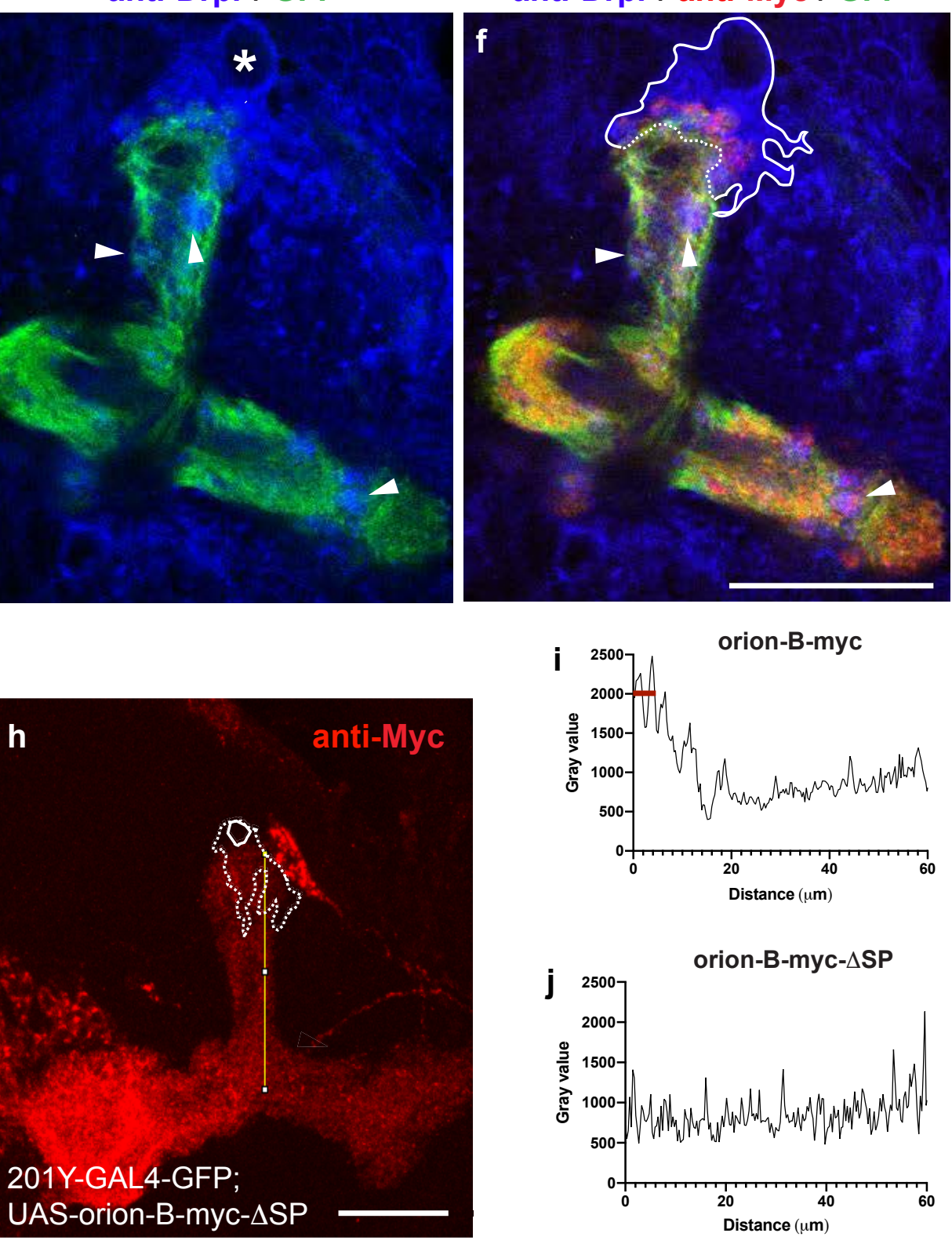

(green) in controls (a, b) and orion $^{l}$ (c) focused on the MB dorsal lobe ( $\mathrm{n}=12 \mathrm{MBs}$ controls and $\mathrm{n}=20 \mathrm{MBs}_{\text {orion }^{l}}$ ). Anti-Fas2 staining (red) reveals spherical hole-like structures occupied

691 by glial processes infiltrating into the $\gamma$ bundle (green, arrows) in wild-type (a, b) but not in orion $^{l}$ individuals (c). Scale bars are $20 \mu \mathrm{m}$. d-f, A single confocal plane showing the expression of $201 Y-G A L 4$ driven $U A S-m C D 8-G F P$ (green, d-f) and Orion-B-Myc (red, d, f) in $6 \mathrm{~h}$ APF MB. Anti-Drpr antibody (blue) was used to visualize the glial cells (blue, e, f). d, displays Orion-B-Myc expression outside of the axons at the top of the vertical $\gamma$ bundle (arrows) as well as in hole-like structures (arrowheads). e, displays an astrocyte positioned at the top of the $\gamma$ bundle (asterisk in its nuclei) as well as several astrocyte processes occupying hole-like structures (arrowheads). Note the co-localization of Orion-B-Myc and glia processes in the hole-like structures (arrowheads in $\mathbf{f}$ ). The astrocyte cell membrane (continuous line) and the membrane contacting the tip of the $\gamma$ bundle (dotted line), where phagocytosis is taking place, based on our interpretation of the astrocyte limits according to the green and the blue channels for GFP and Drpr, respectively, are indicated in f. Scale bar is $40 \mu \mathrm{m} . \mathbf{g}, \mathbf{h}$, Representative images to illustrate how the quantitation of Orion-B-Myc expression (red), $\mathbf{g}$, and Orion-B- $\triangle$ SP-Myc, $\mathbf{h}$, driven by $201 Y$-GAL4 in a traced $60 \mu \mathrm{m}$ line contained in a $\gamma$ axon vertical bundle was performed. The position of an astrocyte (dotted line), labeled by anti-Drpr staining (not shown), and its nucleus (solid circle) are indicated. i, j Representative plotted intensity profiles of Orion expression (gray value) in Orion-B-Myc- (i) or Orion-B- $\Delta$ SP-Mycexpressing MBs $(\mathbf{j})$, according to the distance from the tips $(0 \mu \mathrm{m})$ to the bottoms $(60 \mu \mathrm{m})$ of vertical $\gamma$ bundles. The highest peaks are always located at less than $7 \mu \mathrm{m}$ distance to the tip of the vertical bundle (red bar) when Orion-B-Myc is expressed $(n=10)$ although this was never the case $(n=9)$ when secretion-deficient Orion-B- $\Delta$ SP-Myc expression was quantified. Scale bar in $\mathbf{g}$ and $\mathbf{h}$ are $30 \mu \mathrm{m}$. Full genotypes are listed in Supplementary list of fly strains. 


\section{Supplementary Materials for}

722

723

Axon-secreted chemokine-like Orion is a signal for astrocyte infiltration

724 during neuronal remodeling

725

726 Ana Boulanger ${ }^{*}$, Camille Thinat ${ }^{1}$, Stephan Züchner ${ }^{2}$, Lee G. Fradkin ${ }^{3}$, Hugues Lortat-Jacob ${ }^{4}$

727 and Jean-Maurice Dura ${ }^{1 *}$

728

729

730 *e-mail: ana.boulanger@igh.cnrs.fr; jean-maurice.dura@igh.cnrs.fr

731

732

733

734 Supplementary Fig. 1 to 10

735 Supplementary list of fly strains

736 
Supplementary Fig. 1
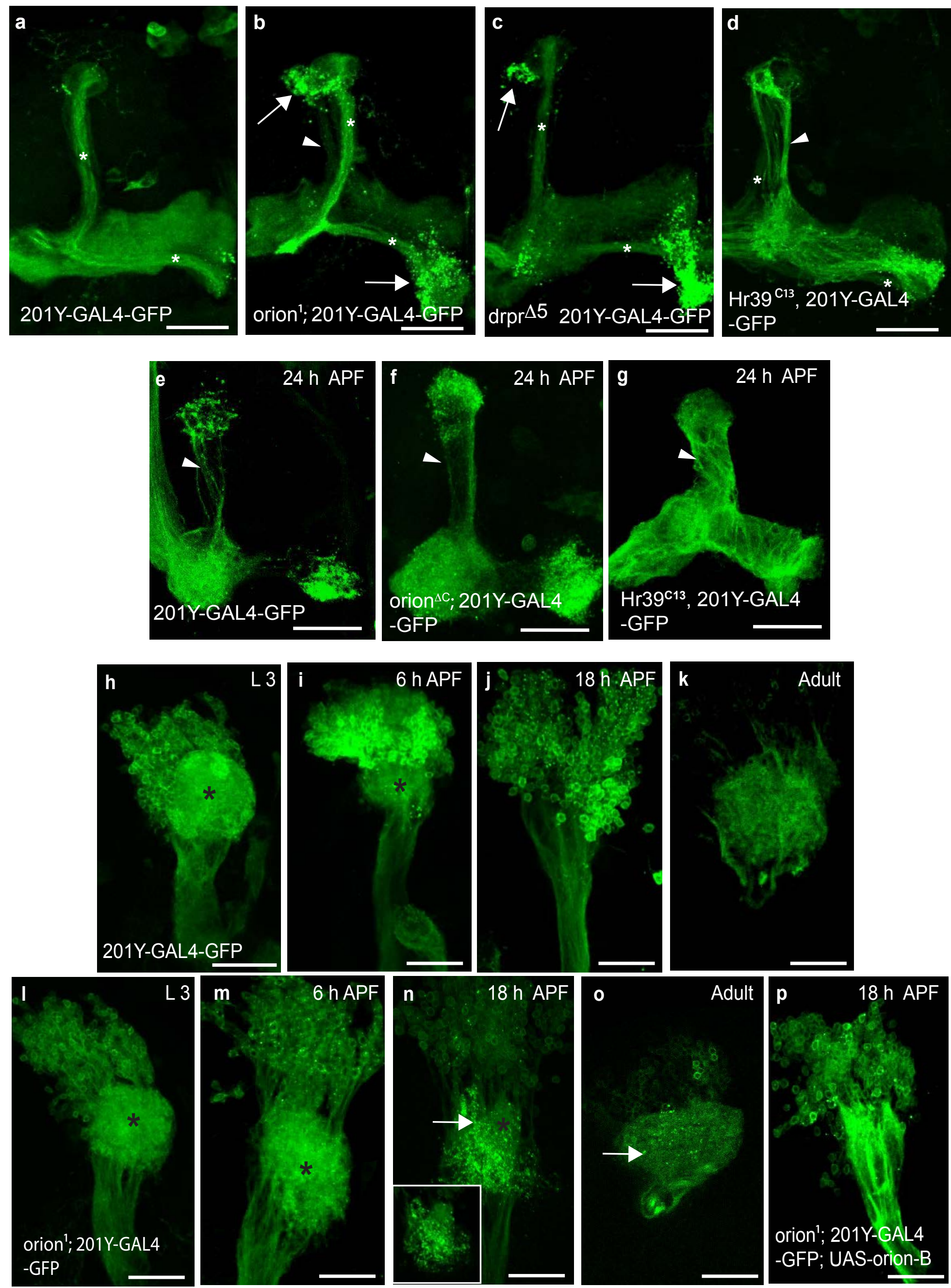

$6 \mathrm{~h} \mathrm{APF}$
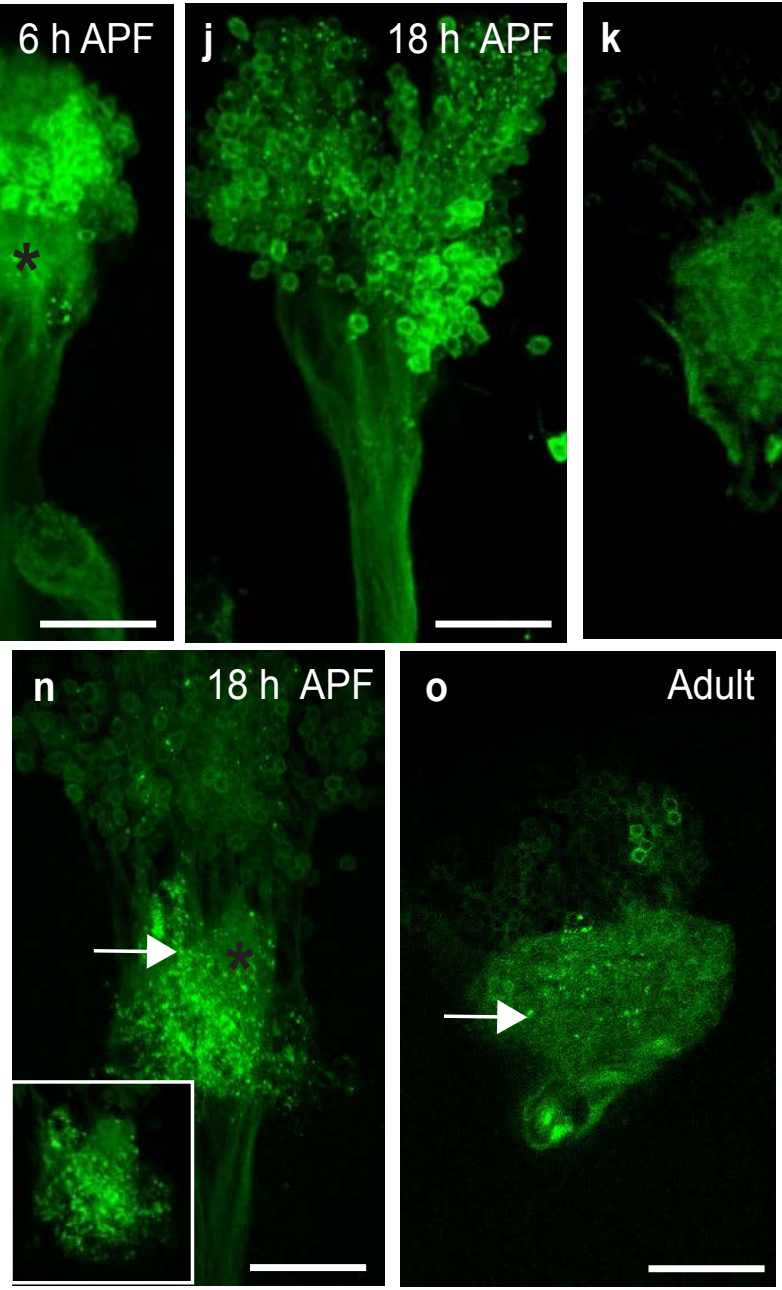

Adult

orion'; 201Y-GAL4 -GFP; UAS-orion-B 
Supplementary Fig. 1. The orion gene is necessary for $\gamma$ axon and dendritic remodeling. a-d, Expression of 201Y-GAL4-driven $U A S$ - $m C D 8$-GFP (green) in adult MB $\gamma$ axons. In adults, this GAL4 line also labels the $\alpha \beta$-core axons indicated here by asterisks. Wild-type (a), orion ${ }^{1}$ (b), $\operatorname{draper}^{\Delta 5}$ (c) and ${\mathrm{Hr} 39^{\mathrm{Cl} 3}}$ (d) $(\mathrm{n}=22,26,30$ and $4 \mathrm{MBs})$. The glial phagocytic receptor Drpr is required for MB remodeling ${ }^{13}$. Note the significant similarity between the orion $^{1}$ and draper $^{\Delta 5}$ phenotypes with respect to the distribution and the amount of axonal debris remaining (arrows in $\mathbf{b}$ and $\mathbf{c}$ ); they differ by the presence of unpruned $\gamma$ axons only in orion $^{1}$ (arrowhead in b; see also Fig. 1b). In addition, the $\operatorname{draper}^{\Delta 5}$ phenotype is observed only in very young flies ${ }^{13}$. In contrast, the orion ${ }^{1}$ phenotype persists throughout adult life at least up to one month old in orion $^{l}$ males. Expression of $\mathrm{Hr} 39$ in $\gamma$ neurons results in only unpruned $\gamma$ axons (arrowhead) without debris (d). In this case, the pruning process is completely blocked due to the $E c R-B 1$ down-regulation by Hr39 thus precluding the generation of axon debris ${ }^{41}$. e-g, Expression of 201Y-GAL4-driven UAS-mCD8-GFP (green) in $\gamma$ neuron axons at $24 \mathrm{~h}$ APF. $\gamma$ axon development was observed in wild-type (e), $\operatorname{orion}^{\Delta C}$ (f) and $\mathrm{Hr} 39^{C l 3}$ (g) as indicated. In wildtype (e), only some scattered $\gamma$ axons are still unpruned (arrowhead). Additional unpruned fascicles of axons (arrowhead) are apparent in orion $^{\Delta C}$ (compare $\mathbf{f}$ with e). Note the massive presence of unpruned $\gamma$ axons (arrowhead) in $\mathrm{Hr}_{3} 9^{\mathrm{Cl3}}$ (g), where the $\gamma$ axon-intrinsic

754 fragmentation process is blocked. However, since the axon-intrinsic fragmentation process is still functional in orion $^{\triangle C}$, the number of these unpruned axons is much lower than in $\mathrm{Hr} 39^{\mathrm{Cl} 3}$

756 ( $\mathrm{n} \geq 10 \mathrm{MBs}$ for each developmental stage). h-p, Expression of 201Y-GAL4-driven UAS$757 m C D 8-G F P$ (green) in $\gamma$ neuron dendrites (black asterisks) during development. Wild-type 758 control (h-k) and orion $^{l}$ (l-p) $\gamma$ dendrites are compared at L3, $6 \mathrm{~h} \mathrm{APF,} 18 \mathrm{~h} \mathrm{APF}$ and adult as indicated. Note the presence of intact larval $\gamma$ dendrites in orion $^{1}$ (asterisk in $\mathbf{n}$ compared to 760 wild-type (j) at $18 \mathrm{~h} \mathrm{APF}$ and the persistence of dendrite debris in orion $^{1}$ at $18 \mathrm{~h} \mathrm{APF} \mathrm{(arrow} \mathrm{in}$ $761 \mathbf{n}$ as well as in adult (arrow in o). A confocal plane of a dendrite region containing larval 762 dendrite debris (brilliant dots) is enclosed by a rectangle in $\mathbf{n}$. p, The orion $^{l}$ unpruned dendritic 763 phenotype is rescued by expression of $U A S$-orion- $B$ at $18 \mathrm{~h} \mathrm{APF}$. All the pictures are confocal 764 Z-projections ( $\mathrm{n}$ is $\geq 8$ for each developmental stage). Scale bars are $40 \mu \mathrm{m}$. Genotypes are listed in Supplementary list of fly strains. 


\section{Presence of unpruned axons in $\geq$ one-week-old adults}

a

\begin{tabular}{|l|r|l|r|l|}
\hline & MB & None & Weak & Strong \\
\hline WT & 25 & 25 & 0 & 0 \\
\hline Hr39 & 22 & 0 & 0 & 22 \\
\hline orion $\Delta C$ & 22 & 0 & 22 & 0 \\
\hline orion1 & 20 & 0 & 20 & 0 \\
\hline orionRNAi & 34 & 0 & 34 & 0 \\
\hline drpr $\Delta 5$ & 22 & 20 & 2 & 0 \\
\hline
\end{tabular}

None

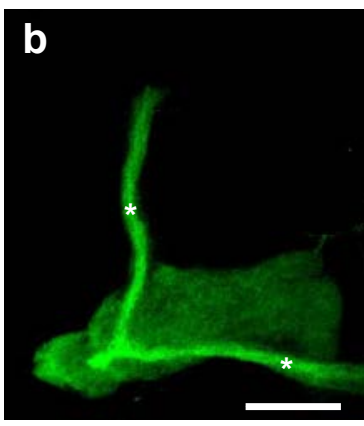

Weak

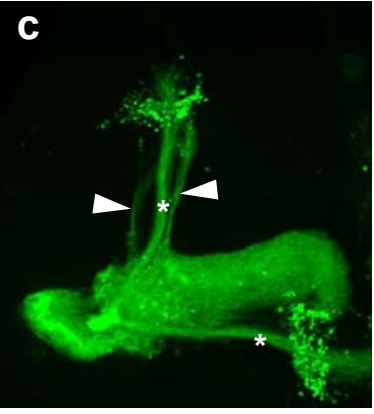

Strong

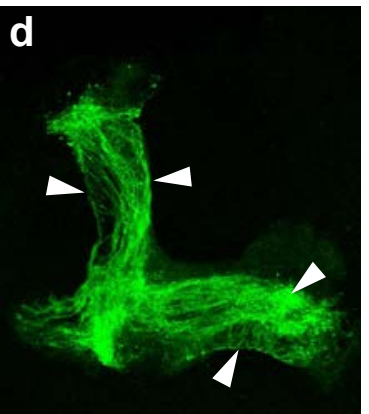

\section{Presence of axon debris in $\geq$ one-week-old adults}

e

\begin{tabular}{|c|c|c|c|c|c|c|}
\hline & MB & None & Mild & Intermediary & Strong & \\
\hline$\overline{W T}$ & 25 & 25 & 0 & 0 & & 0 \\
\hline $\mathrm{Hr} 39$ & 22 & 22 & 0 & 0 & & 0 \\
\hline orion $\Delta C$ & 22 & 0 & 0 & 0 & & 22 \\
\hline orion1 & 20 & 0 & 0 & 0 & & 20 \\
\hline orionRNAi & 34 & 34 & 0 & 0 & & 0 \\
\hline $\mathrm{drpr} \Delta 5$ & 22 & 16 & 2 & 2 & & 2 \\
\hline
\end{tabular}

None

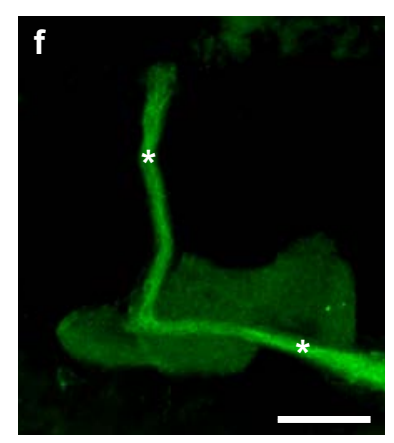

Mild

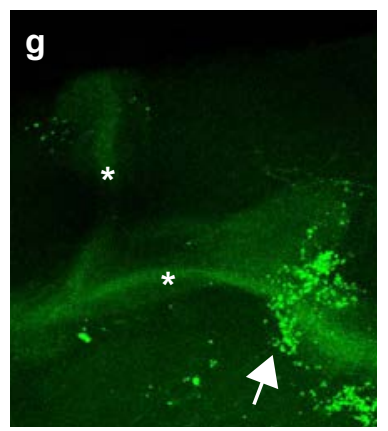

Intermediary

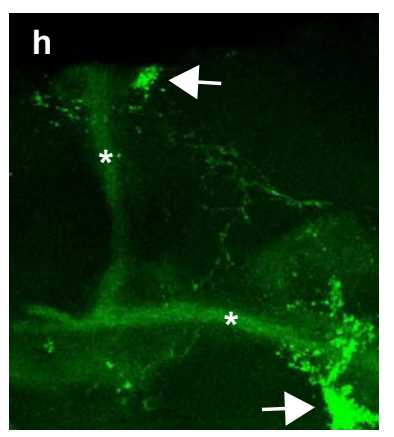

Strong

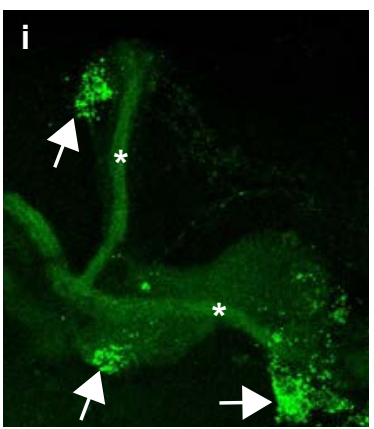

Presence of axon debris in $\leq \mathbf{2} \mathrm{h}$-old adults

$$
\text { j }
$$

\begin{tabular}{|l|r|r|r|r|r|}
\hline & MB & Scattered dots & \multicolumn{1}{|c|}{ Mild } & Intermediary & Strong \\
\hline WT & 10 & 10 & 0 & 0 & 0 \\
\hline orion $\Delta C$ & 12 & 0 & 0 & 0 & 12 \\
\hline $\operatorname{drpr} \Delta 5$ & 73 & 40 & 11 & 4 & 18 \\
\hline
\end{tabular}

None (scattered dots)

Mild

Intermediary

Strong
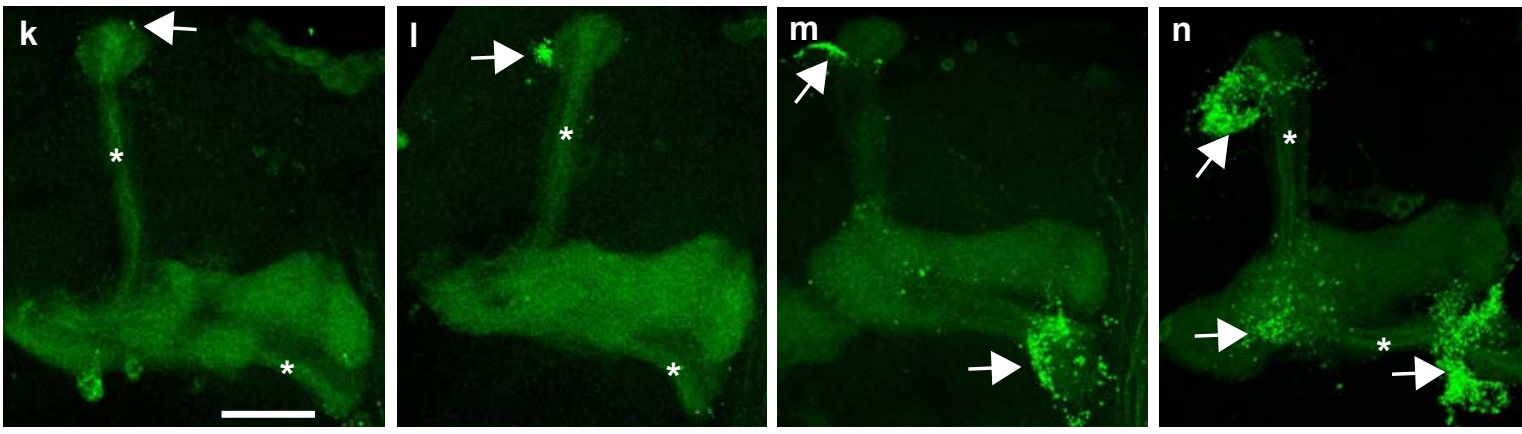
767 Supplementary Fig. 2. Unpruned axons and axon debris phenotypes. Tables a, e and j show 768 quantitation of the unpruned axon (a) and axon debris (e, $\mathbf{j})$ and are described in Table I. $\gamma$ 769 neurons are visualized by the expression of $201 Y$-GAL4 driven UAS-mCD8-GFP (green). In 770 adults, this GAL4 line also labels the $\alpha \beta$-core axons indicated here by asterisks. Unpruned 771 axons are labeled by arrowheads in c ("Weak") and in $\mathbf{d}$ ("Strong"). Axon debris are ranked as 772 "Scattered dots" (k), "Mild" (g, l), "Intermediate" $(\mathbf{h}, \mathbf{m})$ and "Strong" (i, $\mathbf{n})$ and are labelled 773 by arrows in $\mathbf{g - n}$. These dots likely correspond to yet uncleared axon debris $(\mathbf{j}, \mathbf{k})$. Scale bars 774 are $30 \mu \mathrm{m}$. Genotypes are listed in Supplementary list of fly strains. 


\section{Supplementary Fig.3}
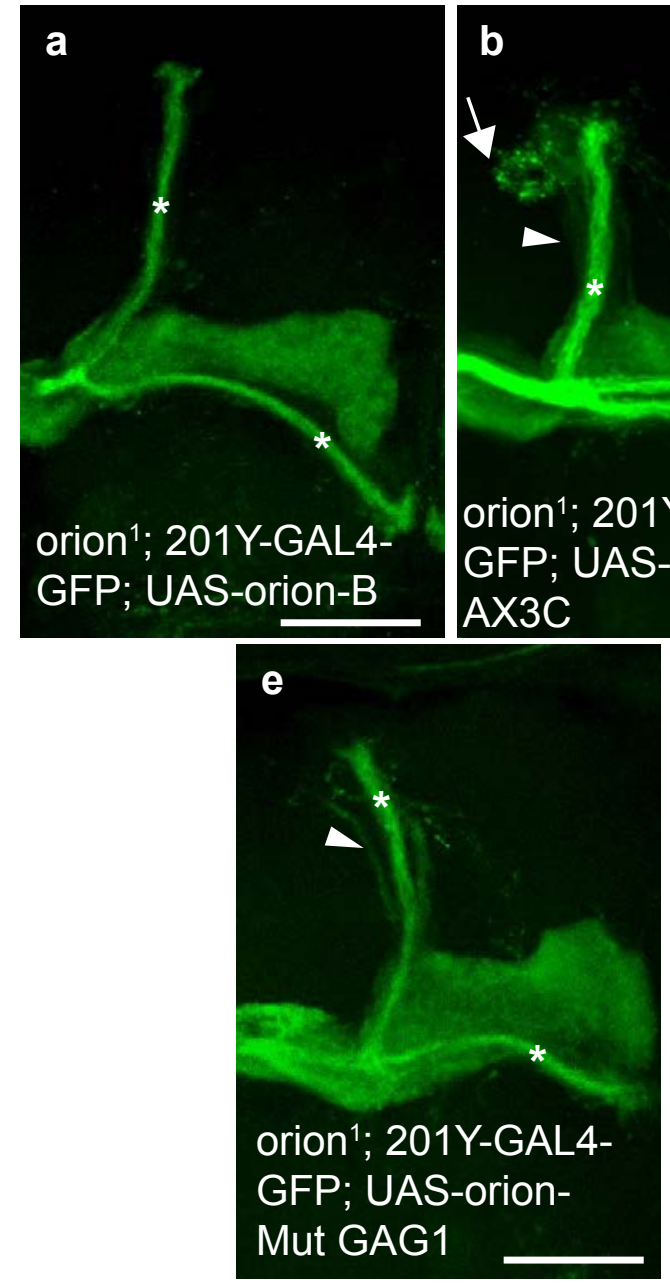

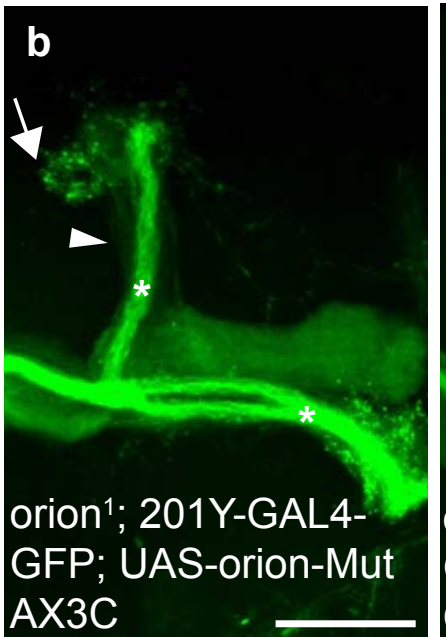

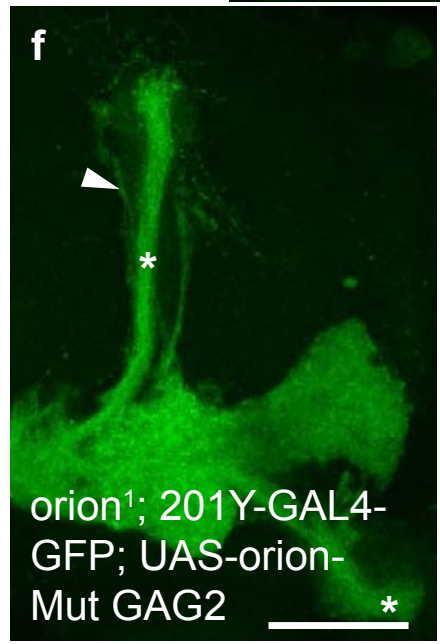
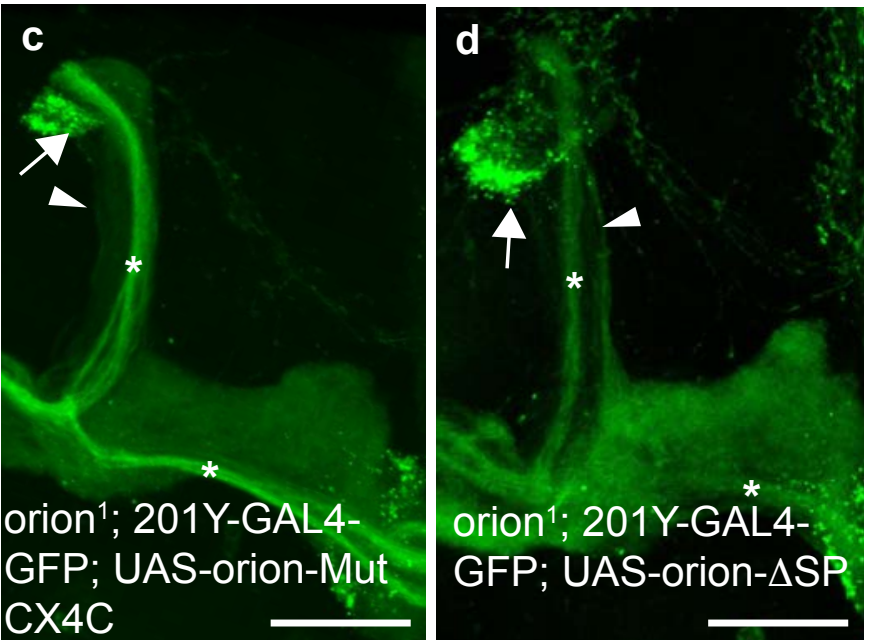

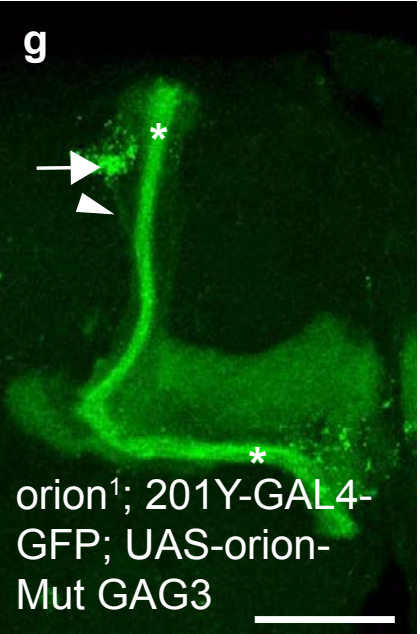

h

\begin{tabular}{|l|r|r|r|r|}
\hline & MB & UP & lebris & WT \\
\hline control & 100 & 0 & 0 & 100 \\
\hline orion1 & 100 & 100 & 100 & 0 \\
\hline orion1 + orion-B WT & 387 & 0 & 0 & 387 \\
\hline orion1 + $\mathrm{SP}$ & 27 & 27 & 27 & 0 \\
\hline orion1 + AX3C & 20 & 20 & 20 & 0 \\
\hline orion1 + CX4C & 20 & 20 & 20 & 0 \\
\hline orion1 + GAG1 & 106 & 46 & 0 & 60 \\
\hline orion1 + GAG2 & 122 & 52 & 0 & 70 \\
\hline orion1 + GAG3 & 118 & 112 & 112 & 6 \\
\hline orion-RNAi & 34 & 34 & 0 & 0 \\
\hline orion-RNAi + EcRB1 & 26 & 2 & 0 & 24 \\
\hline orion-RNAi + control & 20 & 20 & 0 & 0 \\
\hline
\end{tabular}

i
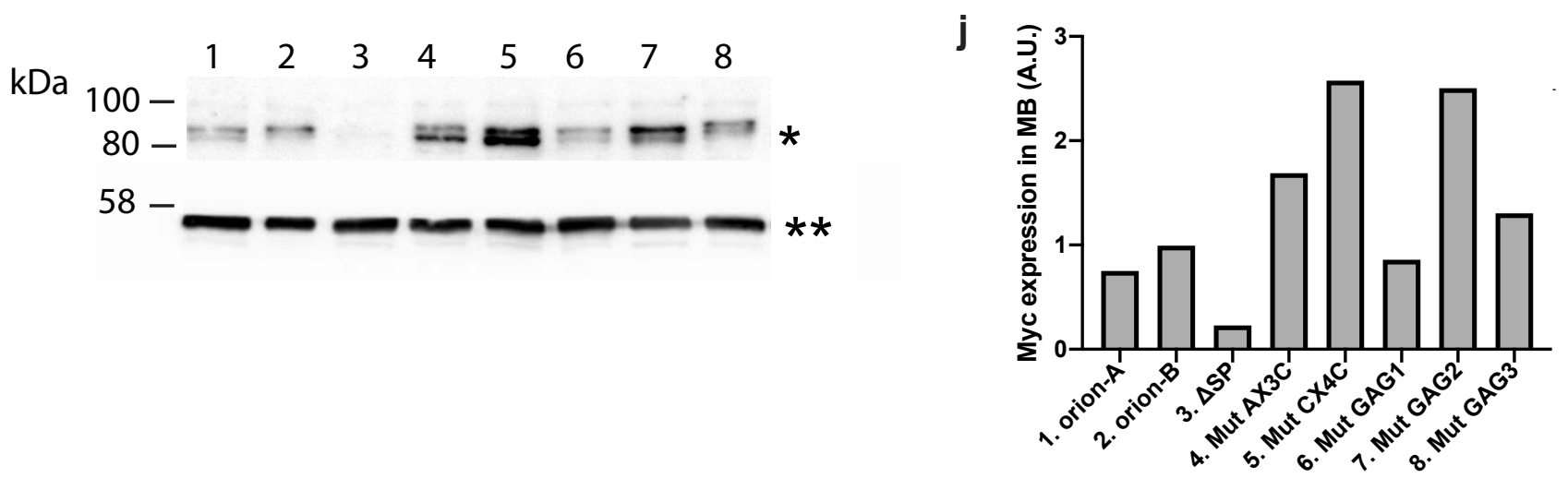
776 Supplementary Fig. 3. The $\mathrm{CX}_{3} \mathrm{C}$ motif, the GAGs sites and the SP domain are required 777 for the Orion pruning function. a-g, The expression of $201 Y$-GAL4 driven UAS-mCD8-GFP 778 (green) is shown in adult MBs in which expression of wild-type UAS-orion-B (a) (n = 387 779 MBs) or UAS-orion-B containing different mutations (Mut, b-g) was induced in orion ${ }^{1}$. UAS780 orion- $B$ contains each of the following mutations: at the $\mathrm{CX}_{3} \mathrm{C}$ site $\left(\mathrm{AX}_{3} \mathrm{C}\right.$ in $\mathbf{b}, \mathrm{n}=20 \mathrm{MBs}$; $781 \mathrm{CX}_{4} \mathrm{C}$ in $\left.\mathbf{c}, \mathrm{n}=20 \mathrm{MBs}\right)$, absence of signal peptide ( $\Delta \mathrm{SP}$ in $\left.\mathbf{d}, \mathrm{n}=27 \mathrm{MBs}\right)$, at the GAG1 site 782 (EKRTERTLKILKD into EAATEATLAILAD in $\mathbf{e}, \mathrm{n}=106 \mathrm{MBs}$ ), at the GAG2 site 783 (VKRNRV into VAANAV in $\mathbf{f}, \mathrm{n}=122 \mathrm{MBs}$ ), at the GAG3 site (ARREKLRL into 784 AAAEALAL in $\mathbf{g}, \mathrm{n}=118 \mathrm{MBs}$ ). Unpruned $\gamma$ axons are labelled by arrowheads, uncleared 785 debris are labelled by arrows and $\alpha \beta$ core axons are labeled by asterisks. Note that debris are 786 absent in e and f. Scale bars are $40 \mu \mathrm{m}$. h, Quantitation of the phenotypes are shown. MB: total 787 number of MBs analyzed; UP: number of MBs containing unpruned $\gamma$ axons; Debris: number 788 of MBs containing uncleared debris; WT: number of wild-type looking MBs. Genotypes are 789 listed in Supplementary list of fly strains. i, Western blot, incubated with an anti-Myc antibody, 790 displaying the Orion-Myc expression levels (single asterisk) produced by the different $U A S$ 791 orion-myc constructs driven by 201Y-GAL4 and the tubulin levels in each genotype (double 792 asterisk) as a control. Proteins were extracted from L3 brains. Lane 1: orion- $A$; lane 2: orion$793 B$; lane 3: orion-B- $\triangle S P$; lane 4: orion-B-Mut $A X 3 C$; line 5: orion-B-Mut $C X 4 C$; line 6: orion794 B-Mut GAG1; lane 7: orion-B-Mut GAG2; lane 8: orion-B-Mut GAG3. j, Orion-Myc band 795 expression levels are shown in arbitrary units (A.U.) which are calculated as a ratio of the Myc 796 level to the loading control tubulin level for each genotype. Note that all of the proteins are 797 expressed at similar or higher levels relative to Orion-B except Orion- $\Delta \mathrm{SP}$ whose expression is 798 lower likely due to protein destabilization resulting from the lack of the signal peptide. 


\section{Supplementary Fig.4}

A

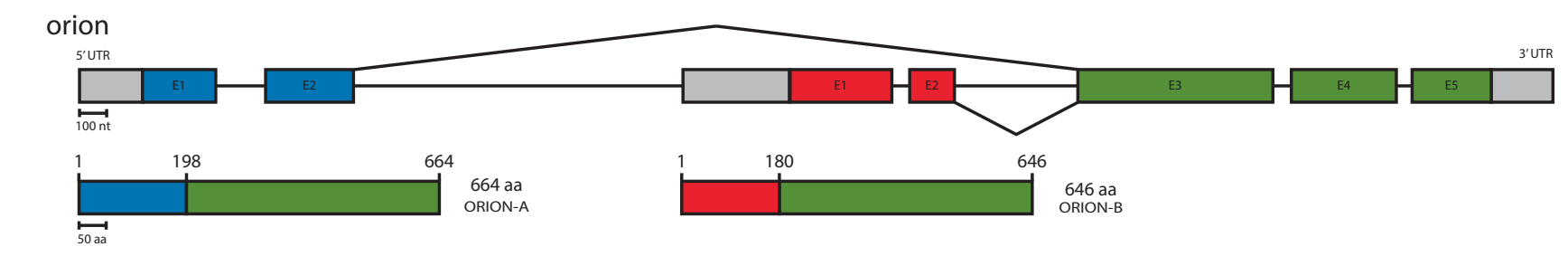

orion $^{\triangle A}$
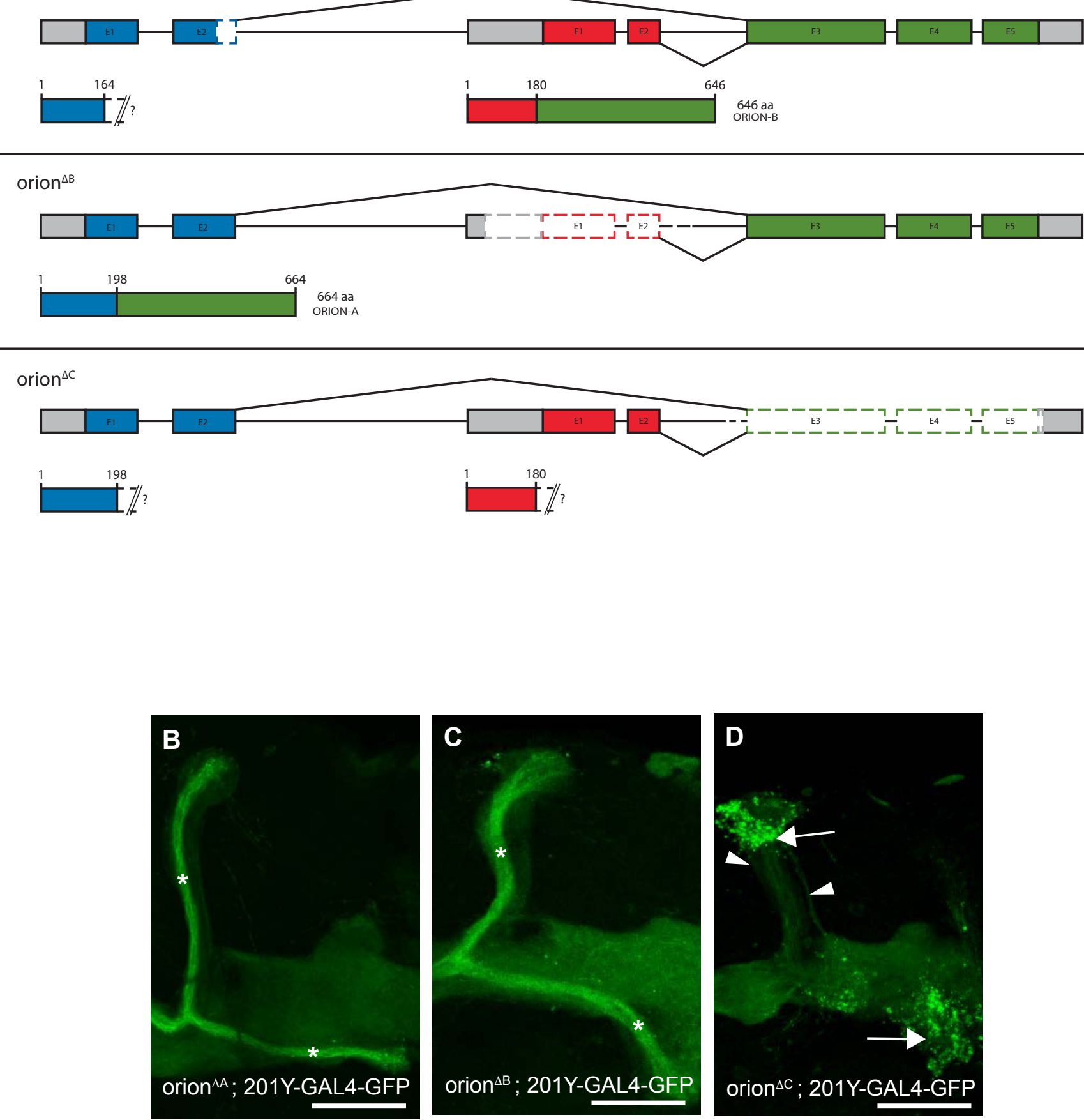
800 Supplementary Fig. 4. Deletion of the common region of orion induces a $\gamma$ axon pruning

801 phenotype. The orion gene (CG2206) lies within the large intron of Ubr3 and is referenced as

802 l(1)G0193 although the lethality is clearly not due to the lack of orion function (see text) but

803 likely to some splicing defect of the Ubr3 mRNA induced by the insertion of transposable

804 elements. a, Schematic representation of orion genomic DNA and its two Orion isoforms in

805 wild-type and in the three different CRISPR induced orion deletions $(\Delta \mathrm{A}, \Delta \mathrm{B}$ and $\Delta \mathrm{C})$ and their

806 corresponding Orion isoforms. b-d, Confocal Z-projections of adult MB are revealed by $201 Y$ -

807 GAL4-driven UAS-mCD8-GFP expression (green) in the three orion CRISPR mutants: orion $^{\triangle A}$,

808 orion $^{\Delta B}$ and orion $^{\Delta C}\left(\mathrm{n}=87,70\right.$ and $98 \mathrm{MBs}$ respectively). Only orion $^{\Delta C}$ displays an unpruned

$809 \gamma$ axon mutant phenotype characterized by unfragmented $\gamma$ axons (arrowhead) and uncleared

810 debris (arrow). $\alpha \beta$ core axons are labeled by asterisks in $\mathbf{b}$ and $\mathbf{c}$ where they are clearly

811 discernible. Scale bars are $40 \mu \mathrm{m}$. Genotypes are listed in Supplementary list of fly strains. 


\section{Supplementary Fig.5}
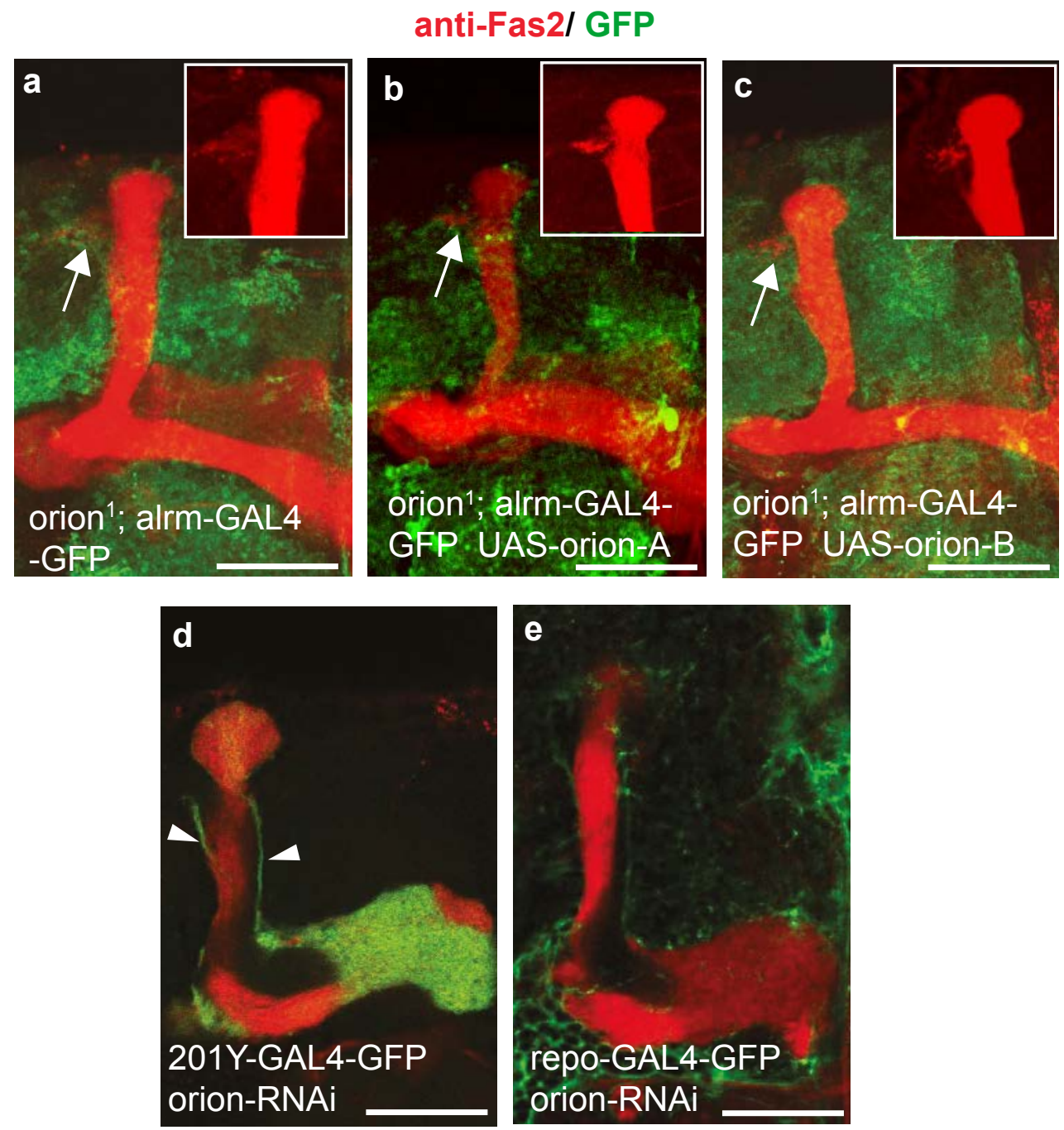


\section{Supplementary Fig. 5. Expression of Orion in glia does not rescue the orion ${ }^{1}$ pruning} 815 defect and downregulating Orion expression in wild-type glia does not affect pruning. a-

816 c, Confocal Z-projections showing merged alrm-GAL4-driven UAS-mCD8-GFP (green) and 817 anti-Fas2 staining (red) in orion $^{1}$ adult MBs. Expression of orion- $A$ (b) or orion- $B$ (c) in 818 astrocytes (alrm-GAL4) does not rescue the orion $^{1}$ mutant phenotype. A rectangle containing 819 anti-Fas2 staining (red) is shown in a-c. Arrows point to unpruned $\gamma$ axons and debris labelled 820 by anti-Fas2 (a-c). d, Confocal plane showing 201Y-GAL4-driven UAS-mCD8-GFP (green) 821 and anti-Fas2 staining (red) in adult MBs. Expression of an UAS-orion-RNAi in MB neurons 822 results in an unpruned $\gamma$ axon phenotype (arrowheads). e, Confocal plane showing repo-GAL4823 driven $U A S-m C D 8-G F P$ (green) and anti-Fas2 staining (red) in adult MBs. Expression of orion824 RNAi in glia does not result in unpruned $\gamma$ axon phenotypes. Scale bars are $40 \mu \mathrm{m}$ and number 825 of MBs is $\geq 20(a, n=20 ; b, n=20 ; c, n=30 ; d, n=20 ; e, n=36$ MBs). Genotypes are listed 826 in Supplementary list of fly strains. 


\section{Supplementary Fig.6}

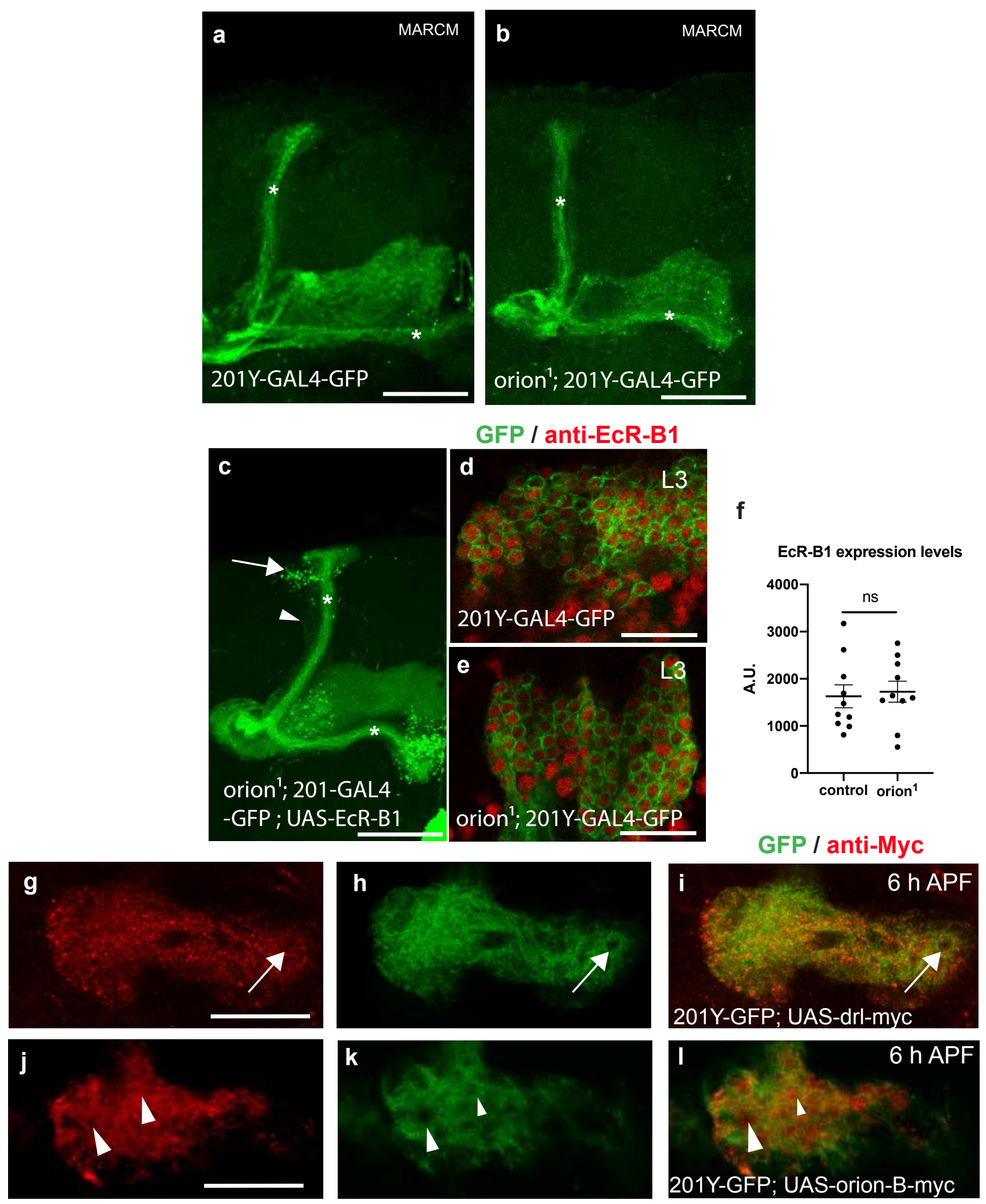


828 Supplementary Fig. 6. Orion is a secreted protein with a non-cell-autonomous function in

$829 \gamma$ axons. a-l, The expression of 201 Y-GAL4 driven UAS-GFP (green) reveals $\gamma$ neurons. In adult

830 (a-c), L3 (d, e) and 6 h APF (g-l). a, b, MARCM neuroblast clones displaying wild-type $\gamma$ axon

831 pruning are shown. A wild-type control (a) and an orion $^{l}(\mathbf{b})\left(\mathrm{n}=20\right.$ wild-type and 30 orion $^{1}$

832 neuroblast clones). c, EcR-B1 expression in orion $^{1} \gamma$ neurons does not rescue the orion ${ }^{1}$

833 phenotype. Note the presence of $\gamma$ remnant debris (arrow) and unpruned axons (arrowhead) (n

$834=40 \mathrm{MBs}$ ). $\mathbf{d}$, e, Mushroom body cell body region showing ECR-B1 expression (red staining)

835 in wild-type (d) and orion $^{1}$ (e). f, Quantitation of EcR-B1 signal in $\gamma$ neuron cell bodies in

836 arbitrary units (A.U.) reveals no significant differences between control and orion $^{1}(\mathrm{n}=10 \mathrm{MBs}$

837 for control and for orion $^{l} ; \mathrm{p}=0.67$ (Mann-Whitney $U$ test)). These interaction analyses support

838 orion being genetically downstream of $E c R-B 1$. g-l, Expression of the transmembrane receptor

$839 d r l-m y c(\mathbf{g}-\mathbf{i}, \mathrm{n}=10)$ and orion-B-myc $(\mathbf{j}-\mathbf{l}, \mathrm{n}=10)$ in MBs. Red represents anti-myc staining.

840 Drl-myc staining is absent in hole-like structures (arrows in g-i). However secreted Orion-myc

841 is present in these structures (arrowheads in $\mathbf{j}-\mathbf{I}$ ). Images are confocal Z-projections, except for

842 g-I which are confocal planes. Scale bars are $40 \mu \mathrm{m}$ in a-c and $\mathbf{g - l}$ and $20 \mu \mathrm{m}$ in $\mathbf{d}$ and e.

843 Genotypes are listed in Supplementary list of fly strains. 
bioRxiv preprint doi: https://doi.org/10.1101/2020.11.23.394049; this version posted November 23, 2020. The copyright holder for this

preprint (which was not certified by peer review) is the author/funder. All rights reserved. No reuse allowed without permission.

Supplementary Fig.7

I(1)G0193

a



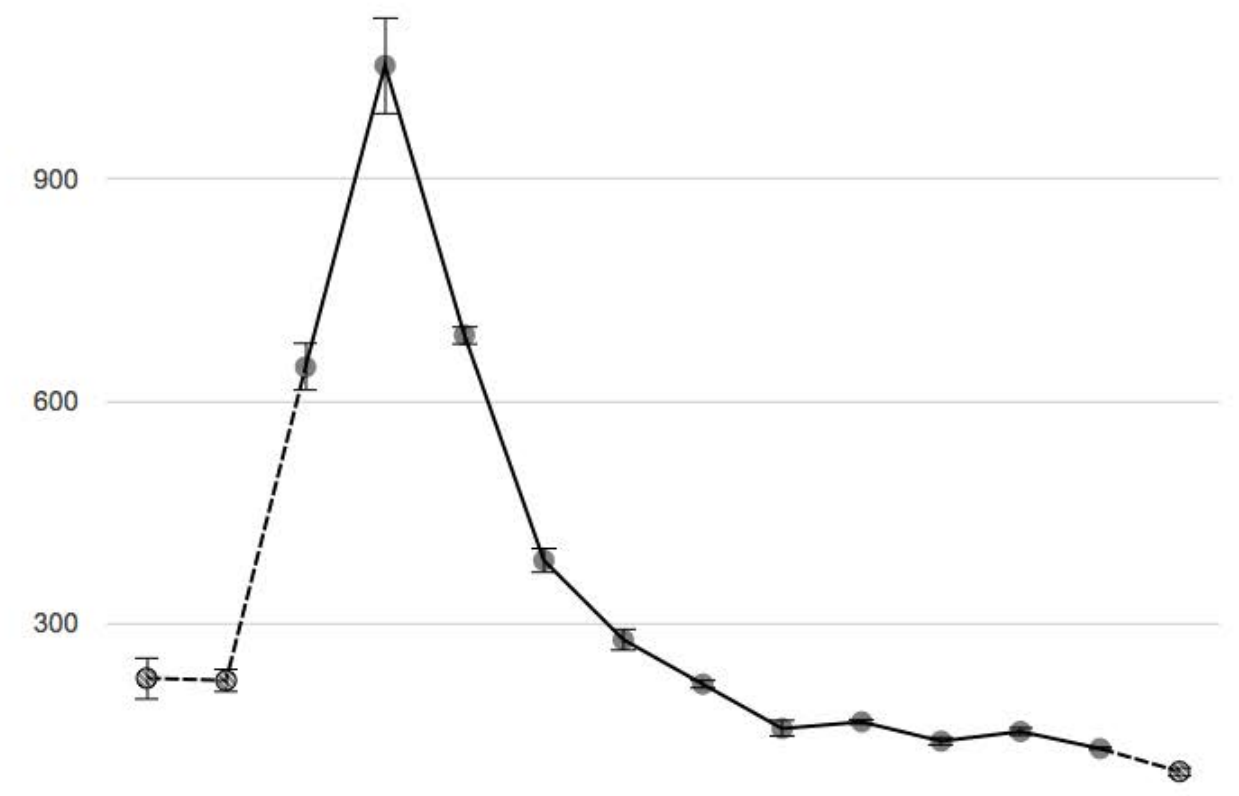

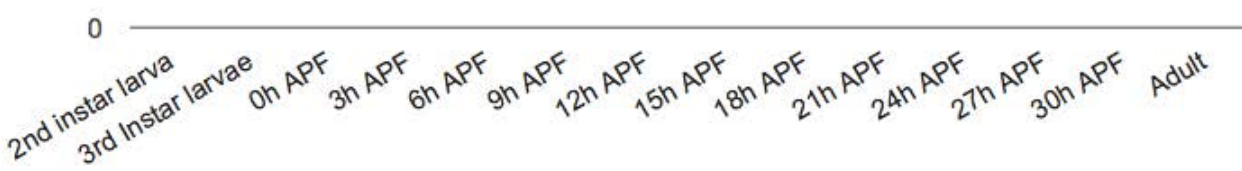

I(1)G0193

b
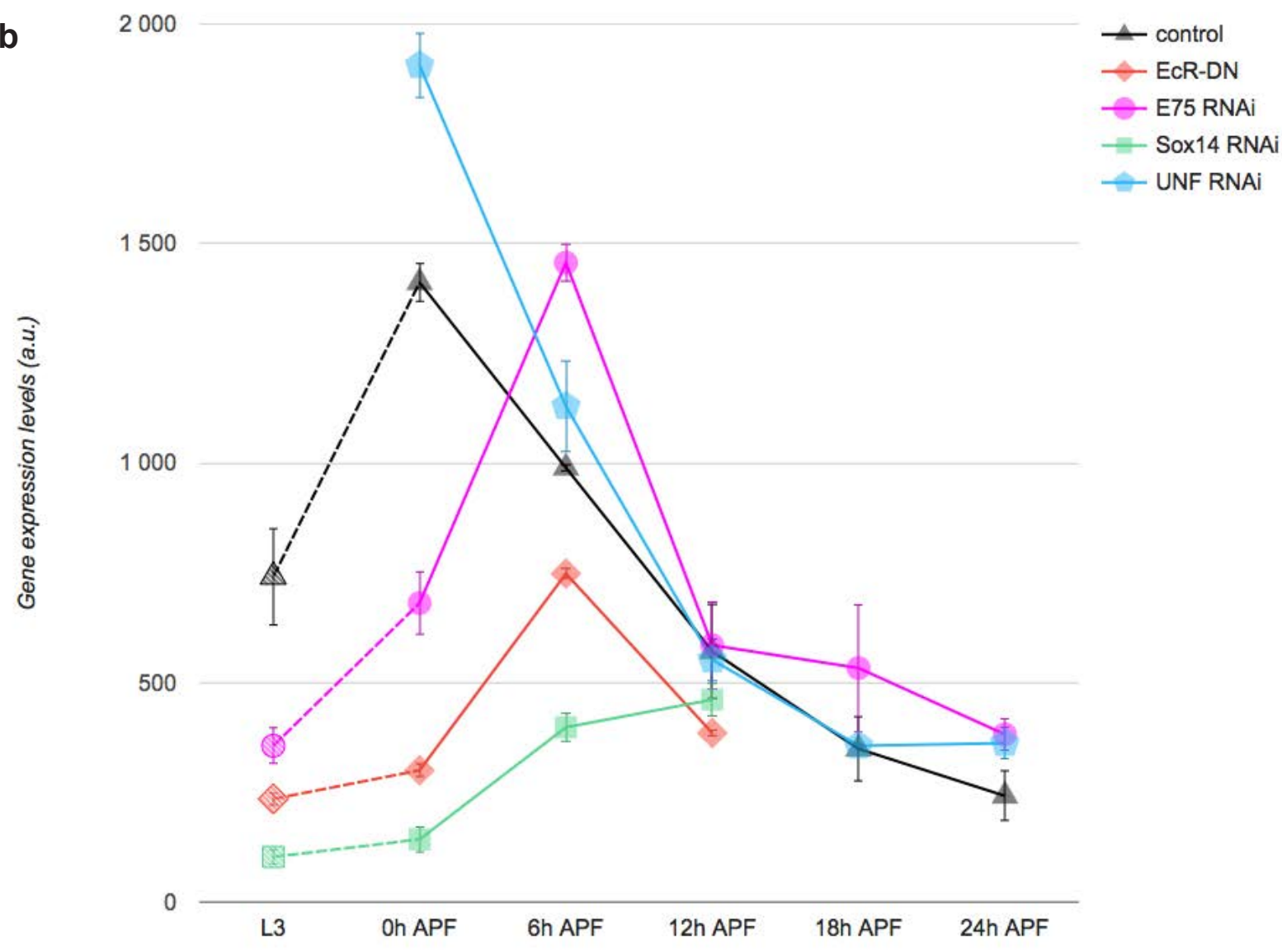

(From Oren Schuldiner's laboratory public web site : https://www.weizmann.ac.il/mcb/Schuldiner/resources) 


\section{Supplementary Fig. 7. orion mRNA is expressed at the time of pruning in $\gamma$ neurons and}

845 its expression is EcR-B1 regulated. a, b, Data showing orion mRNA $\gamma$ neuron-expression

846 levels in arbitrary units (a.u.) during development in wild-type (a) and in different mutant

847 backgrounds (b), downloaded from Oren Schuldiner's laboratory's public web site 848 (http://www.weizmann.ac.il/mcb/Schuldiner/resources) ${ }^{24}$. Note that the peak of expression of

849 orion is at $3 \mathrm{~h} \mathrm{APF}$ which is the timepoint at which pruning initiates (a). We also note that just 850 before and during the pruning process (0-6 h APF) orion $m R N A$ expression (black line in b) is 851 regulated by EcR-B1 and Sox 14. 
bioRxiv preprint doi: https://doi.org/10.1101/2020.11.23.394049; this version posted November 23, 2020. The copyright holder for this

preprint (which was not certified by peer review) is the author/funder. All rights reserved. No reuse allowed without permission.

Supplementary Fig.8

anti-Fas2 / GFP
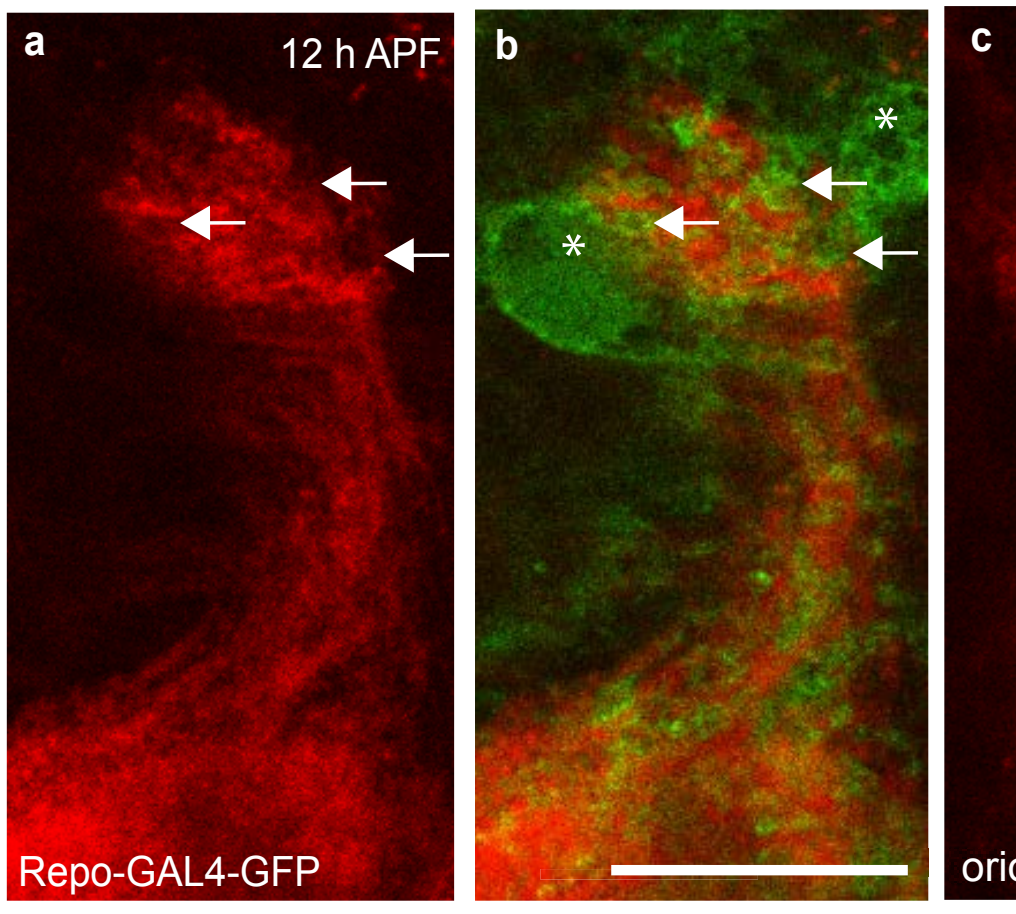

12 h APF

d

orion $^{1}$; repo-GAL4-GFP

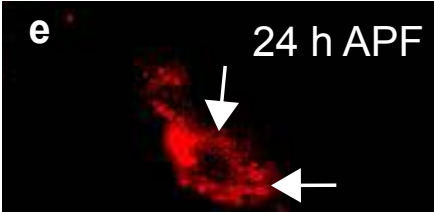

Repo-GAL4-GFP
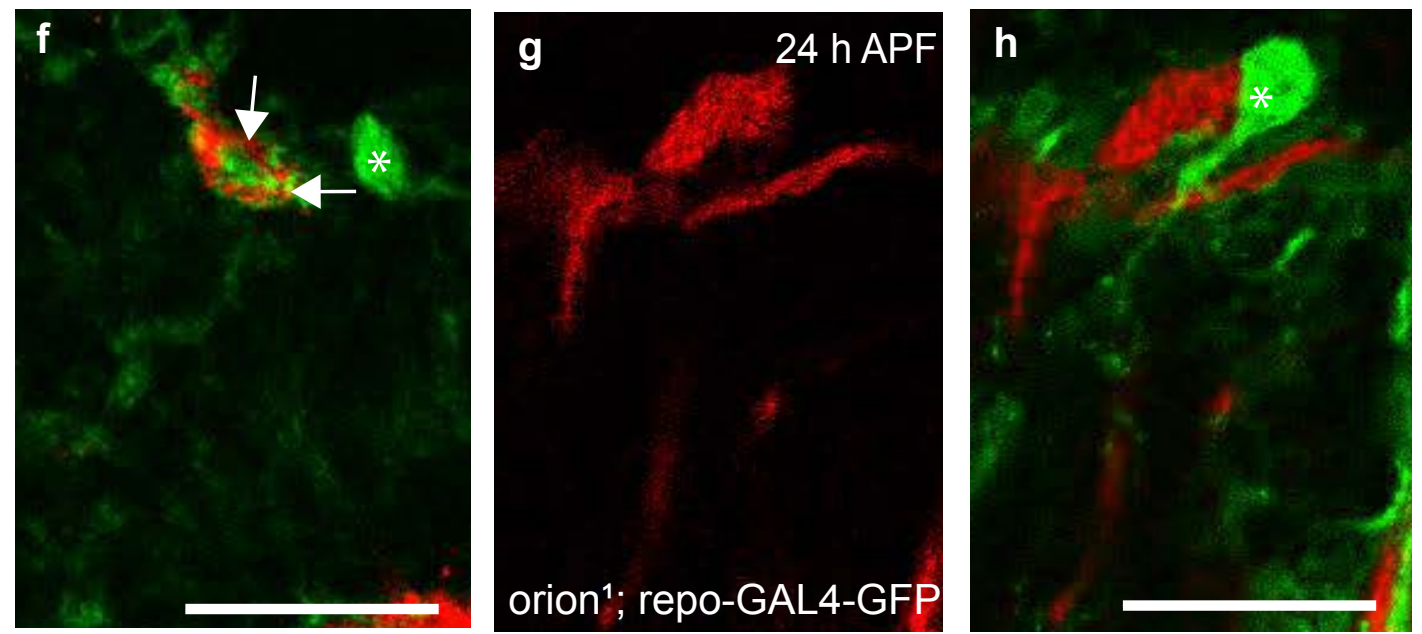

\section{anti-Fas2 / anti-REPO / GFP}

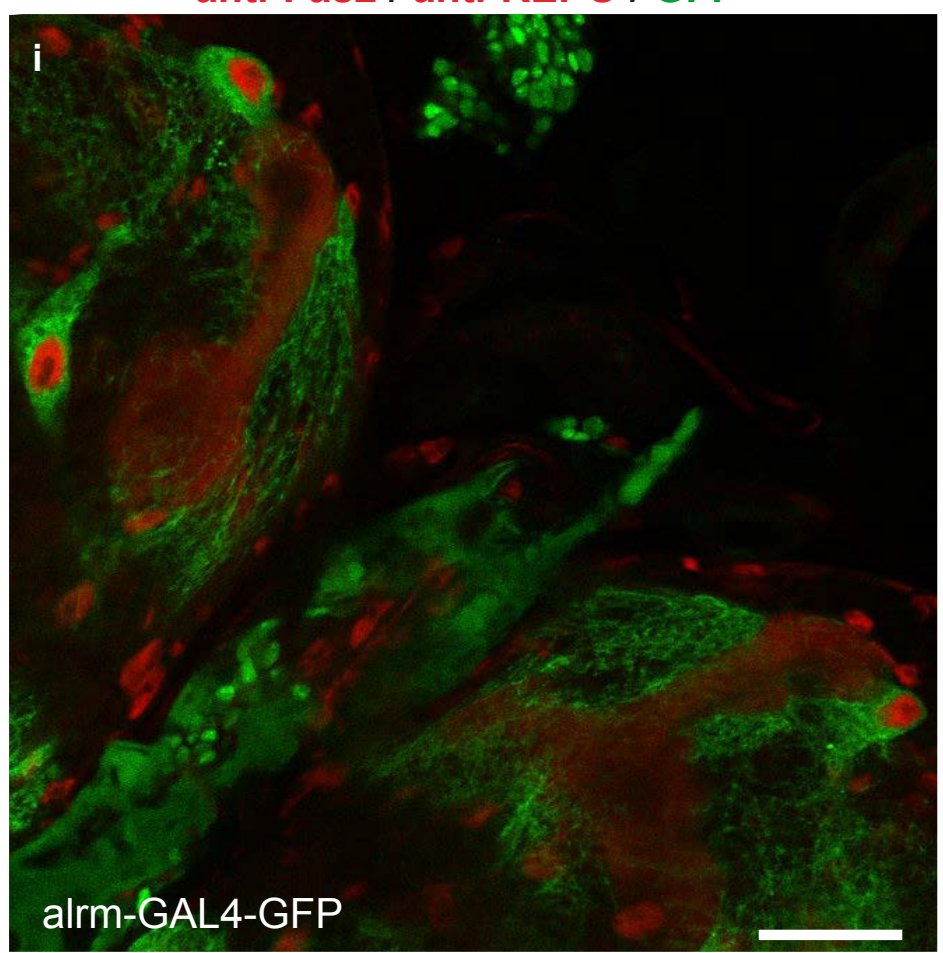

j

Astrocyte number

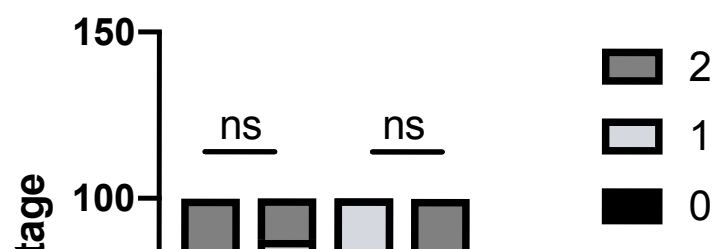


853 Supplementary Fig. 8. Orion is required for the infiltration of astrocytes into the MB $\gamma$

854 bundle during development and its mutation does not alter the number of astrocytes 855 surrounding the $\gamma$ axons.

$856 \mathbf{a}-\mathbf{h}$, Confocal Z-projections of $12 \mathrm{~h}$ and $24 \mathrm{~h}$ APF brains expressing repo-GAL4-driven UAS$857 m C D 8-G F P$ (green) in controls (a, b for $12 \mathrm{~h} \mathrm{APF} \mathrm{and} \mathbf{e}, \mathbf{f}$ for $24 \mathrm{~h} \mathrm{APF}$ ) and orion $^{1}$ (c, $\mathbf{d}$ for $85812 \mathrm{~h} \mathrm{APF}$ and $\mathbf{g}, \mathbf{h}$ for $24 \mathrm{~h} \mathrm{APF})$ focused on the MB dorsal lobe $(\mathrm{n}=10$ control MBs and $\mathrm{n}=$ 85910 orion $^{l} \mathrm{MBs}$ ). Anti-Fas2 staining (red) reveals spherical hole-like structures occupied by glial 860 processes infiltrating into the $\gamma$ bundle (green, arrows) in wild-type (a, b and e, f) but not in 861 orion $^{1}$ individuals (c, $\mathbf{d}$ and $\mathbf{g}, \mathbf{h}$ ). Note the significant infiltration of the $\gamma$ bundle by two 862 astrocytes in $\mathbf{b}$ (asterisk) and the absence of axon bundle infiltration by a closely apposed astrocyte in $\mathbf{d}$ (asterisk). Nevertheless, the global aspect of the $\gamma$ bundle where the fragmentation

864 is taking place looks similar in wild-type and mutant at $12 \mathrm{~h} \mathrm{APF}$. This suggests that, in orion 865 mutant, fragmenting $\gamma$ axons are not actively being engulfed by astrocytes. Scale bars are 20 $866 \mu \mathrm{m}$. i, Confocal Z-projection showing UAS-mCD8-GFP expression (green) in astrocytes driven 867 by alrm-GAL4 at L3. Red shows both glial cell nuclei labelled by an anti-Repo antibody and 868 MBs labelled by anti-Fas2. Scale bars are $40 \mu \mathrm{m}$. j, Percentage of astrocytes surrounding the $\gamma$ 869 vertical lobe at L3 and at $6 \mathrm{~h}$ APF in wild-type and orion $^{l}$ (for L3, n = 24 wild-type MBs and n $870=16$ orion $^{l} \mathrm{MBs}$; for $6 \mathrm{~h} \mathrm{APF,} \mathrm{n}=20$ wild-type MBs and $\mathrm{n}=18$ orion $^{1} \mathrm{MBs}$ ). No statistically871 significant differences were observed between the two groups (Fisher's exact test $p=0.84$ for $872 \mathrm{~L} 3$ and $\mathrm{p}=0.12$ for $6 \mathrm{~h} \mathrm{APF}$ ). Scale bar is $30 \mu \mathrm{m}$. Genotypes are listed in Supplementary list 873 of fly strains. 


\section{Supplementary Fig.9}
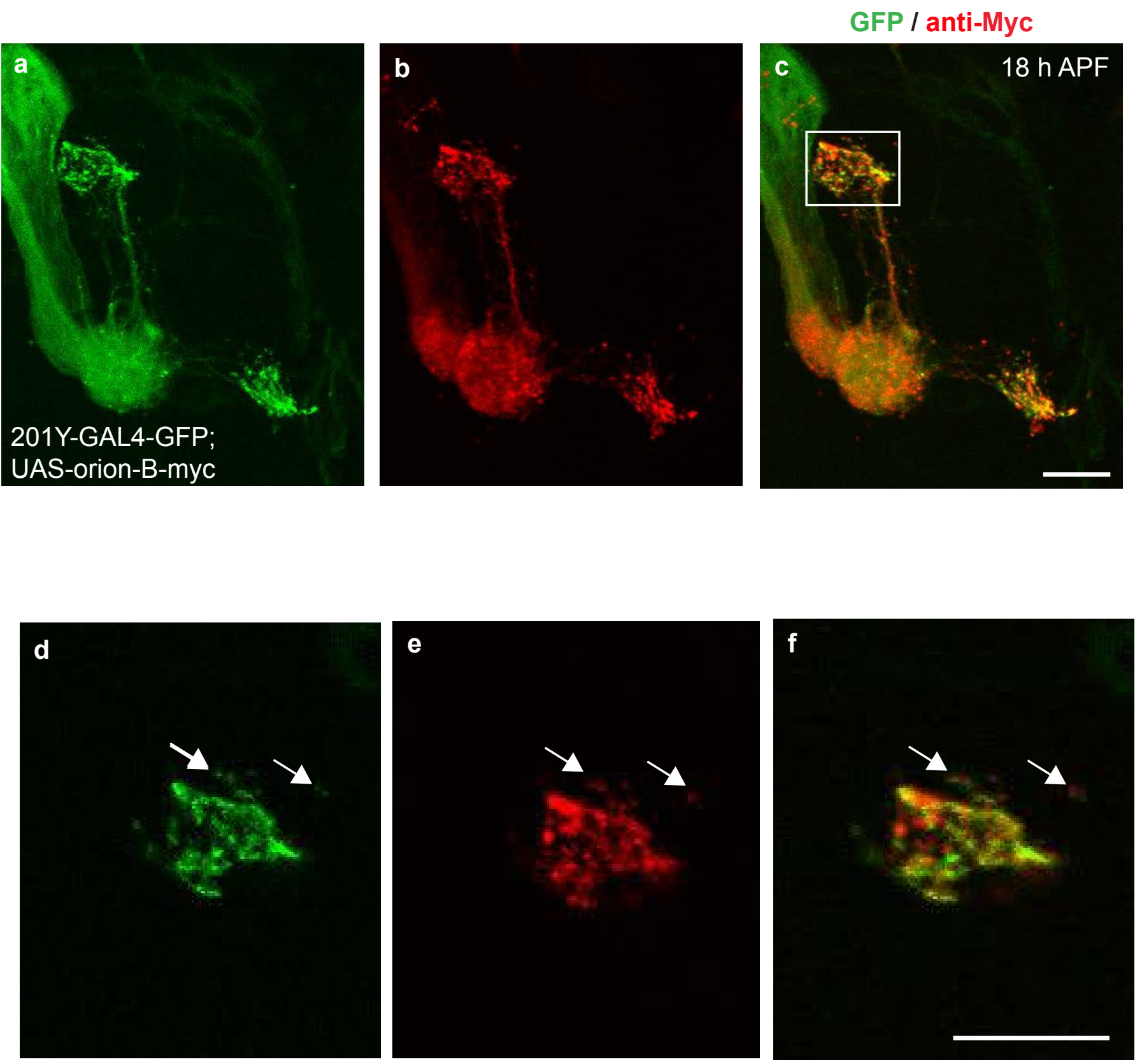
879 Supplementary Fig. 9. Orion is associated with membranes. a-c, Expression of $U A S-m C D 8-$ $880 G F P$ (green) and UAS-orion-B-myc (red) under the control of $201 Y$-GAL4 is shown in $18 \mathrm{~h}$ 881 APF $\gamma$ neurons $(n=6)$. d-f, (confocal planes) are higher magnifications of the a-c (confocal Z882 projections) regions enclosed by rectangles. Some debris staining for both GFP and Orion-Myc 883 is labelled by arrows. Scale bars are $20 \mu \mathrm{m}$. Genotypes are listed in Supplementary list of fly 884 strains. 


\section{Supplementary Fig.10}

\section{anti-Drpr / GFP}

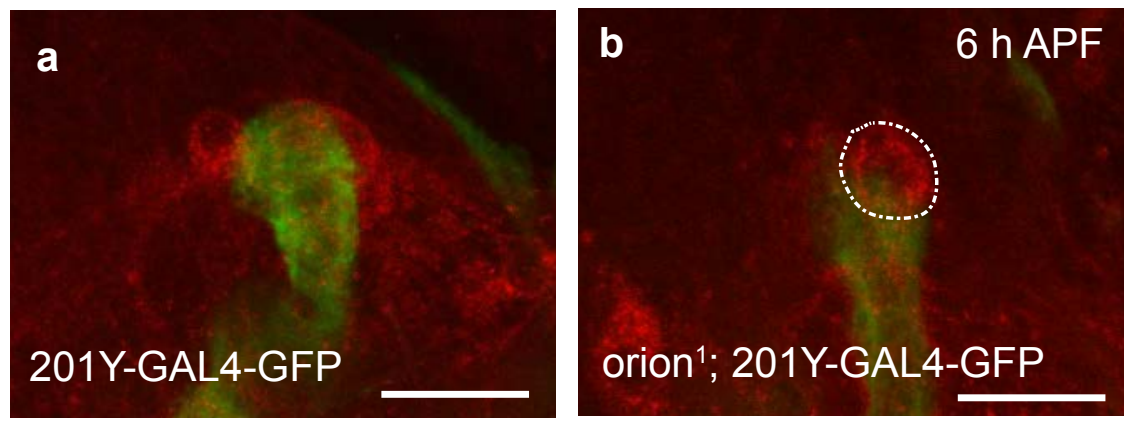

anti-Fas2 / anti-Repo / GFP
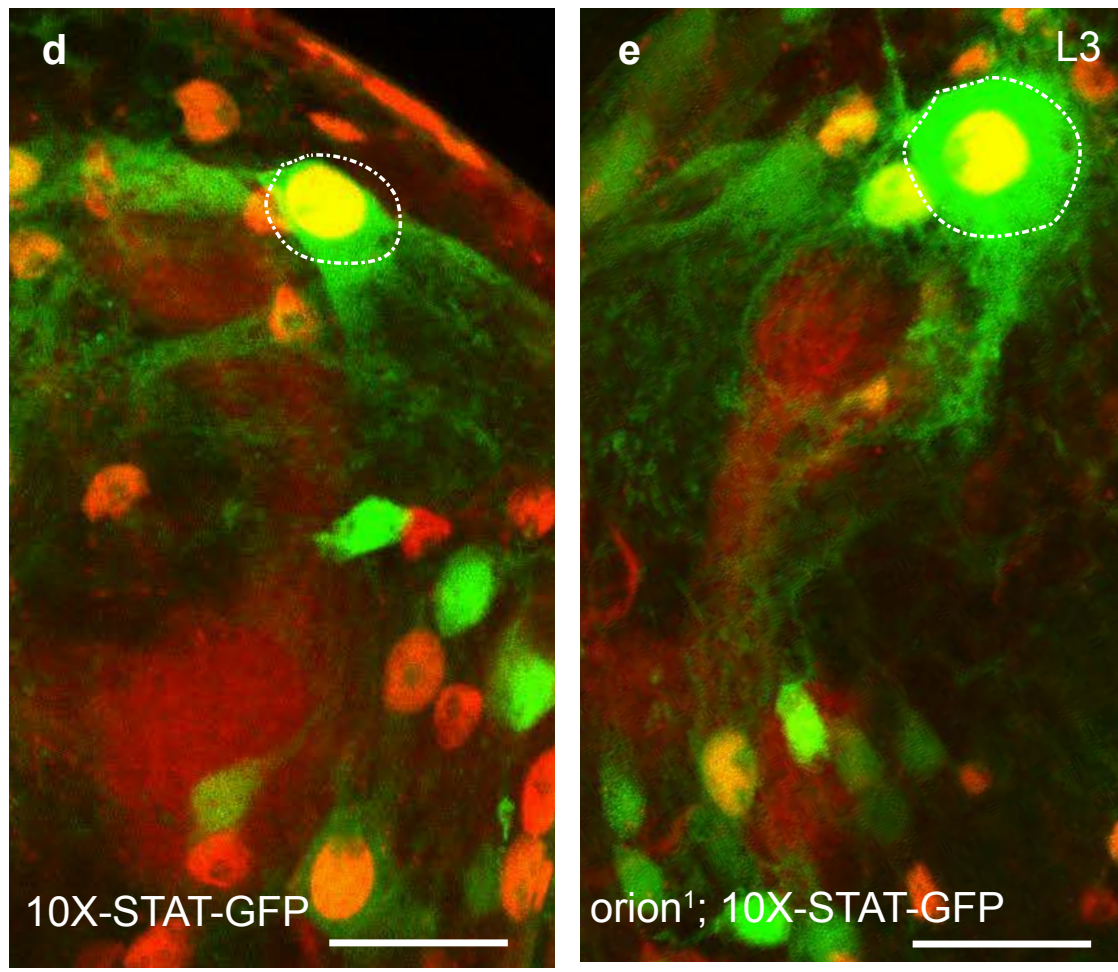

C

Drpr expression levels

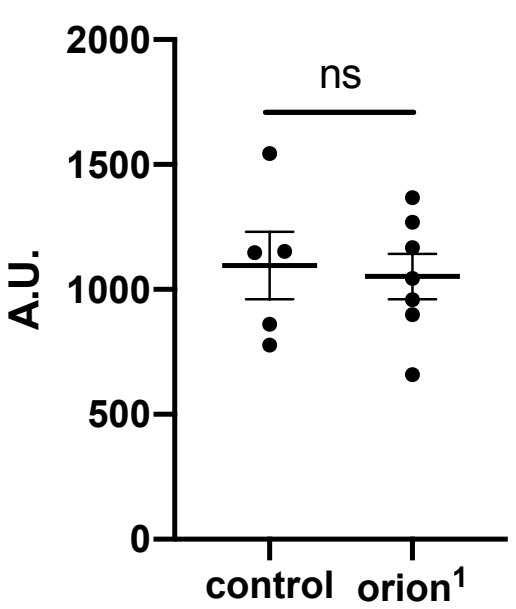

f

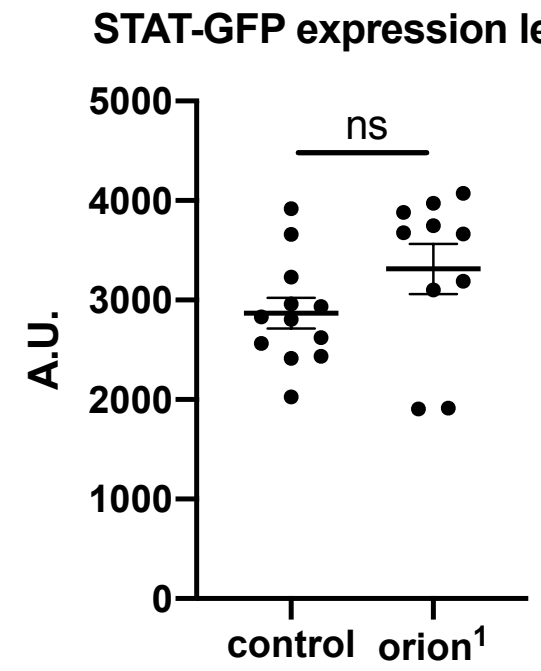


885 Supplementary Fig. 10. orion $^{1}$ mutants display wild-type levels of Drpr protein expression 886 and wild-type activity of the $d r p r$ transcriptional regulator STAT92E. Since $d r p r$ 887 expression is regulated by known axon degeneration cues $^{30,45}$ and the $d r p r^{\Delta 5}$ and orion $^{1}$ alleles 888 display mutant phenotypes that share some features (Supplementary Fig.1), we analyzed Drpr 889 expression in wild-type and $\operatorname{orion}^{1}$. a-c, Expression of Drpr (red) in wild-type control (a) and 890 orion $^{1}$ (b) in $6 \mathrm{~h} \mathrm{APF} \mathrm{brains} \mathrm{and} \mathrm{the} \mathrm{corresponding} \mathrm{quantitation} \mathrm{in} \mathrm{arbitrary} \mathrm{units} \mathrm{(A.U.)} \mathrm{in}$ 891 astrocytes (c). Green corresponds to the expression of 201Y-GAL4 driven UAS-mCD8-GFP in $892 \gamma$ axons. The astrocyte cytoplasm, in which quantitation are performed, is circled by a white 893 dotted line in a and b. c, Quantitation of Drpr expression in arbitrary units (A.U.) reveals no 894 significant differences between control and orion $^{l}$. Results are means \pm S.E.M. $\mathrm{n}=5$ MBs for 895 control and $7 \mathrm{MBs}$ for orion $^{1} ; \mathrm{p}=1$ (Mann-Whitney $U$ test). In addition, since $d r p r$ expression 896 is regulated by the transcription factor STAT92E ${ }^{46}$, we analyzed the expression of an STAT92E897 GFP reporter in wild-type and orion $^{1}$. d-f, Expression of 10X-STAT92E-GFP (green) in wild898 type control (d) and orion $^{1}$ (e) larval brains and the corresponding quantitation in arbitrary units 899 (A.U.) (f). Red is both, Fas 2 labelling $\gamma$ axon bundles and Repo antibody labelling the glial cell 900 nuclei. Pictures are confocal Z-projections. The astrocyte cytoplasm, in which quantitation are 901 performed, is circled by a white dotted line in d and e. f, Quantitation of STAT-GFP expression 902 in arbitrary units (A.U.) reveals no significant differences between control and orion ${ }^{1}$. Results 903 are means \pm S.E.M. $\mathrm{n}=12 \mathrm{MBs}$ for control and $10 \mathrm{MBs}$ for orion $^{l} ; \mathrm{p}=0.09$ (Mann-Whitney $904 U$ test). Scale bars are $30 \mu \mathrm{m}$ in $\mathbf{a}, \mathbf{b}$ and $20 \mu \mathrm{m}$ in d, e. Genotypes are listed in Supplementary 905 list of fly strains. 
907

908

909

910

911

912

913

914

915

916

917

918

919

920

921

922

923

924

925

926

927

928

929

930

931

932

933

934

935

936

937

938

939

940

\section{Supplementary list of fly strains:}

Fig. 1: a, c-e, $y w^{67 c 23} / \mathrm{Y}$ or $y w^{67 c 23} / y w^{67 c 23} ; U A S-m C D 8 G F P 201 Y-G A L 4 /+$. b, f-i, $y w^{67 c 23}$ $s n^{3}$ orion ${ }^{1}$ FRT19A / Y or $y w^{67 c 23} s^{3}$ orion ${ }^{1}$ FRT19A / y $w^{67 c 23} \operatorname{sn}^{3}$ orion ${ }^{1}$ FRT19A; UASmCD8GFP 201Y-GAL4 /+. j, y w $w^{67223}$ sn $^{3}$ orion $^{1} F R T 19 A / Y$; UAS-mCD8GFP 201Y-GAL4/+; UAS-orion-A-myc / +. k, y w w7c23 sn $^{3}$ orion $^{1}$ FRT19A / Y; UAS-mCD8GFP 201Y-GAL4 / +; UAS-orion-B-myc / +. 1, $y w^{67 c 23} / Y$ or $y w^{67 c 23} / w^{1118}$; UAS-mCD8GFP 201Y-GAL4 / UASorion-RNAi.

Fig. 3: a, b, d, e, g, h, j, k, $y w^{67 c 23} / \mathrm{Y}$ or $y w^{67 c 23} / y w^{*} ; U A S-m C D 8 G F P 201 Y-G A L 4 /+$; $U A S$-orion-B-myc / +. c, f, i, $y w^{67 c 23} / \mathrm{Y}$ or $y w^{67 c 23} / y w^{*} ; U A S-m C D 8 G F P 201 Y$-GAL4 / + ; $U A S$-orion- $B-\triangle S P-m y c /+$.

Fig. 4: a, b, $y w^{67 c 23} s n^{3} F R T 19 A / Y$; repo-GAL4 UAS-mCD8GFP / +. c, $y w^{67 c 23} s n^{3}$ orion $^{1}$ FRT19A / Y ; repo-GAL4 UAS-mCD8GFP / +. d-g, $y w^{67 c 23} / \mathrm{Y}$ or $y w^{67 c 23} / y w^{*} ; U A S-$ mCD8GFP 201Y-GAL4 / + ; UAS-orion-B-myc / +. h, y w $w^{67 c 23} / \mathrm{Y}$ or $y w^{67 c 23} / y w^{*} ; U A S$ mCD8GFP $201 Y$-GAL4 / + ; UAS-orion-B- $\triangle S P-m y c /+$.

Supplementary Fig. 1: a, e, h-k, $y w^{67 c 23} / Y$ or $y w^{67 c 23} / y w^{67 c 23}$; UAS-mCD8GFP 201Y-GAL4 / +. b, l-o, $y w^{67 c 23} s^{3}$ orion $^{1} F R T 19 A / Y$ or $y w^{67 c 23} s^{3}$ orion $^{1}$ FRT19A / y $w^{67 c 23}$ sn $^{3}$ orion $^{1}$; UAS-mCD8GFP 201Y-GAL4 / +. c, $y w^{67 c 23} / \mathrm{Y}$ or $y w^{67 c 23} / y w^{67 c 23} ; U A S-m C D 8 G F P 201 Y$ $G A L 4 /+; d r p r^{\Delta 5} / d r p r^{\Delta 5}$. d, g, $y w^{67 c 23} / \mathrm{Y}$ or $y w^{67 c 23} / y w^{67 c 23} ; H r 39^{C 13}, U A S-m C D 8 G F P$ $201 Y-G A L 4 /+$ f,$y w^{*}$ orion $^{\Delta C} / \mathrm{Y} ; U A S-m C D 8 G F P 201 Y-G A L 4 /+$ p, y $w^{67 c 23}$ sn $^{3}$ orion $^{1}$ FRT19A /Y ; UAS-mCD8GFP 201Y-GAL4 / + ; UAS-orion-B-myc /+.

Supplementary Fig. 2: a, e and j. WT: $y w^{67 c 23} /$ Y; UAS-mCD8GFP 201Y-GAL4 / +. Hr39: $y w^{67 c 23} / \mathrm{Y} ; H r 39^{C 13}, U A S-m C D 8 G F P 201 Y-G A L 4 /+$ orion $\Delta \mathbf{C}: y w^{*}$ orion $^{\Delta C} / \mathrm{Y} ; U A S$ mCD8GFP 201Y-GAL4 / +. orion1: $y w^{67 c 23} \mathrm{sn}^{3}$ orion $^{1}$ FRT19A /Y ; UAS-mCD8GFP $201 Y$ GAL4 / +. orionRNAi: $y w^{67 c 23} / Y$; UAS-mCD8GFP $201 Y$-GAL4 / UAS-orion-RNAi. drprA5: $y w^{67 c 23} / \mathrm{Y} ; U A S-m C D 8 G F P 201 Y-G A L 4 /+; d r p r^{\Delta 5} / d r p r^{\Delta 5} . \mathbf{b}, \mathbf{f}, y w^{67 c 23} / \mathrm{Y} ; U A S-$ mCD8GFP 201Y-GAL4 /+. c, y w $w^{67 c 23}$ sn $^{3}$ orion $^{1}$ FRT19A / Y ; UAS-mCD8GFP 201Y-GAL4 /+. d: $y w^{67 c 23} / Y$; Hr39 $9^{C 13}, U A S-m C D 8 G F P 201 Y-G A L 4$ /+. g-i, k-m, UAS-mCD8GFP $201 Y$ $G A L 4 /+; d r p r^{\Delta 5} / d r p r^{\Delta 5}$. n, $y w^{*}$ orion $^{\Delta C} / \mathrm{Y} ; U A S-m C D 8 G F P 201 Y-G A L 4 /+$. 
941 Supplementary Fig. 3: a, y w $w^{67 c 23} \mathrm{sn}^{3}$ orion $^{1}$ FRT19A /Y; UAS-mCD8GFP 201Y-GAL4 / +; $942 U A S$-orion-B-myc / +. b, y w $w^{67 c 23}$ sn $^{3}$ orion $^{1}$ FRT19A / Y ; UAS-mCD8GFP 201 Y-GAL4 / +; 943 UAS-orion-B-Mut AX3C-myc / +. c, y w $w^{67 c 23}$ sn $^{3}$ orion $^{1} F R T 19 A / \mathrm{Y} ; U A S-m C D 8 G F P 201 Y$ 944 GAL4 / + ; UAS-orion-B-Mut CX4C-myc- / +. d, y w $w^{67 c 23} \mathrm{sn}^{3}$ orion $^{1}$ FRT19A / Y; UASmCD8GFP 201Y-GAL4 / + ; UAS-orion-B- $\Delta S P$-myc / +. e, y w $w^{67 c 23}$ sn $^{3}$ orion $^{1}$ FRT19A / Y; 946 UAS-mCD8GFP 201Y-GAL4 / +; UAS-orion-B-Mut GAG1-myc / +. f, y w $w^{67 c 23} \mathrm{sn}^{3}$ orion $^{1}$ FRT19A / Y; UAS-mCD8GFP 201Y-GAL4 / +; UAS-orion-B-Mut GAG2-myc / +. g, y w wc23 sn ${ }^{3}$ orion $^{1}$ FRT19A /Y; UAS-mCD8GFP 201 Y-GAL4 / +; UAS-orion-B-Mut GAG3-myc / +. h, control: $y w^{67 c 23} / \mathrm{Y}$; UAS-mCD8GFP 201Y-GAL4 / + orion1: $y w^{67 c 23} \operatorname{sn}^{3}$ orion $^{1}$ FRT19A / Y; UAS-mCD8GFP $201 Y$-GAL4 / + orion1 + orion-B WT: see above (a). orion1 + $\triangle$ SP: see above (d). orion1 + AX3C: see above (b). orion1 + CX4C: see above (c). orion1 + GAG1: see above (e). orion1 + GAG2: see above (f). orion1 + GAG3: see above (g). orion-RNAi: $y$ $w^{67 c 23} / Y$; UAS-mCD8GFP 201Y-GAL4 / UAS-orion-RNAi. orion-RNAi + EcR-B1: $y w^{67 c 23} /$ Y; UAS-mCD8GFP 201Y-GAL4 / UAS-orion-RNAi; UAS-EcR-B1/+. orion-RNAi + control : $y w^{67 c 23} / Y ; \quad U A S-m C D 8 G F P 201 Y$-GAL4 / UAS-orion-RNAi; UAS-FRT-y+-FRT/+. i, $1: y$ $w^{67 c 23}$ sn $^{3}$ orion $^{1}$ FRT19A / Y; UAS-mCD8GFP 201Y-GAL4 / +; UAS-orion-A-myc / +. $2: y$ $w^{67 c 23}$ sn $^{3}$ orion $^{1}$ FRT19A / Y; UAS-mCD8GFP 201Y-GAL4 / +; UAS-orion-B-myc / +. $3: y$ $w^{67 c 23}$ sn $^{3}$ orion $^{1} F R T 19 A / Y$; UAS-mCD8GFP 201Y-GAL4 / + ; UAS-orion-B - $\triangle S P-m y c /+$. $4: y w^{67 c 23}$ sn $^{3}$ orion $^{1}$ FRT19A/Y ; UAS-mCD8GFP 201Y-GAL4 / +; UAS-orion-B-Mut AX3C960 myc / +. $5: y w^{67 c 23}$ sn $^{3}$ orion $^{1}$ FRT19A / Y ;UAS-mCD8GFP 201Y-GAL4 / + ; UAS-orion-BMut CX4C-myc/+. $6: y w^{67 c 23} \mathrm{sn}^{3}$ orion $^{1}$ FRT19A /Y; UAS-mCD8GFP 201Y-GAL4 / +; UASorion-B-Mut GAG1-myc / +. $7: y w^{67 c 23}$ sn $^{3}$ orion $^{1}$ FRT19A /Y; UAS-mCD8GFP 201Y-GAL4 / +; UAS-orion-B -Mut GAG2-myc / +. $8: y w^{67 c 23}$ sn $^{3}$ orion $^{1}$ FRT19A /Y; UAS-mCD8GFP $201 Y$-GAL4 / +; UAS-orion-B -Mut GAG3-myc / +.

Supplementary Fig. 4: b, $y w^{*}$ orion $^{\triangle A} / \mathrm{Y}$; UAS-mCD8GFP 201Y-GAL4 /+. c, $y w^{*}$ orion $^{\Delta B /}$ Y ; UAS-mCD8GFP 201Y-GAL4 / +. d, $y w^{*}$ orion $^{\Delta C} / \mathrm{Y} ; U A S-m C D 8 G F P 201 Y-G A L 4 /+$.

Supplementary Fig. 5: a, y $w^{67 c 23}{s n^{3}}^{o r i o n}{ }^{1} F R T 19 A / Y$; CyO, $P(D f d-G M R-n v Y F P) 2 /+$ or Sp / + ; alrm-GAL4 UAS-mCD8GFP / +. b, y w $w^{67 c 23}$ sn $^{3}$ orion $^{1} F R T 19 A$ / Y ; CyO, P(DfdGMR-nvYFP)2 /+ or Sp/+ ; alrm-GAL4 UAS-mCD8GFP /UAS-orion-A-myc. c, y w ${ }^{67 c 23} s n^{3}$ orion $^{1} F R T 19 A$ / Y ; CyO, P(Dfd-GMR-nvYFP $) 2$ /+ or Sp / + ; alrm-GAL4 UAS-mCD8GFP / UAS-orion-B-myc. d, $y w^{67 c 23} / \mathrm{Y}$ or $y w^{67 c 23} / w^{*} ; U A S-m C D 8 G F P 201 Y$-GAL4 / UAS-orionRNAi. e, $w^{*} / Y$ or $w^{*} / w^{*}$; UAS-orion-RNAi/+; repo-GAL4 UAS-mCD8GFP/+. 
976 Supplementary Fig. 6: a, $w^{*} t u b-P-G A L 80 h s-F L P 122$ FRT19A / y $w^{67 c 23} s n^{3}$ FRT19A ; UASmCD8GFP 201Y-GAL4 / +. b, w* tub-P-GAL80 hs-FLP122 FRT19A / y w $w^{67 c 23}$ sn $^{3}$ orion $^{1}$ FRT19A ; UAS-mCD8GFP 201Y-GAL4/+. c, y w $w^{67 c 23}$ sn $^{3}$ orion $^{1} F R T 19 A / Y$; UAS-mCD8GFP $201 Y-G A L 4 /+$; UAS-EcR-B1 /+. d, $y w^{67 c 23} s n^{3}$ FRT19A / Y ; UAS-mCD8GFP 201Y-GAL4 / +. e, $y w^{67 c 23} s^{3}$ orion $^{1} F R T 19 A / \mathrm{Y}$; UAS-mCD8GFP 201Y-GAL4/+. g-i, $y w^{67 c 23} / \mathrm{Y}$ or $y$ $w^{67 c 23} / y w^{67 c 23} ; U A S-m C D 8 G F P 201 Y-G A L 4 /+; 2 x U A S-d r l-m y c /+. \mathbf{j}-\mathbf{l}, y w^{67 c 23} / \mathrm{Y}$; or $y$ $w^{67 c 23} / y w^{*} \quad U A S-m C D 8 G F P 201 Y-G A L 4 /+; U A S$-orion-B-myc / +

983

984

Supplementary Fig. 8: a, b, e, f, $y w^{67 c 23} s n^{3} F R T 19 A / Y$; repo-GAL4 UAS-mCD8GFP / +. c, d, g, hy $w^{67 c 23} s^{3}$ orion $^{1} F R T 19 A / \mathrm{Y} ;$ repo-GAL4 UAS-mCD8GFP / +. i, w*/Y or $w^{*} /$ $w^{*}$; CyO, P(Dfd-GMR-nvYFP $) 2$ / Sp ; alrm-GAL4 UAS-mCD8GFP / alrm-GAL4 UAS$m C D 8 G F P$. j, WT $: y w^{67 c 23} / Y$; CyO, P(Dfd-GMR-nvYFP $) 2 /+$ or Sp / + ; alrm-GAL4 UASmCD8GFP /+. orion ${ }^{1}: y w^{67 c 23}$ sn $^{3}$ orion $^{1} F R T 19 A / Y ; C y O, P(D f d-G M R-n v Y F P) 2 /+$ or Sp / + ; alrm-GAL4 UAS-mCD8GFP /+.

990

Supplementary Fig. 9: a-f, $y w^{67 c 23} / \mathrm{Y}$ or $y w^{67 c 23} / y w^{*} ; U A S-m C D 8 G F P 201 Y-G A L 4 /+$;

994 Supplementary Fig. 10: a, $y w^{67 c 23} / \mathrm{Y}$ or $y w^{67 c 23} / y w^{67 c 23} ; U A S-m C D 8 G F P$ 201Y-GAL4 /

$995 C y O . \mathbf{b}, y w^{67 c 23} s^{3}$ orion $^{1}$ FRT19A / Y or $y w^{67 c 23}{s n^{3}}^{\text {orion }^{1}}$ FRT19A /y $w^{67 c 23} \mathrm{sn}^{3}$ orion $^{1}$

996 FRT19A; UAS-mCD8GFP 201Y-GAL4 / CyO. d, $y w^{67 c 23} s n^{3}$ FRT19A /Y ; 10X-STAT92E- 